\title{
HotFuzz: Discovering Algorithmic Denial-of-Service Vulnerabilities Through Guided Micro-Fuzzing
}

\author{
William Blair ${ }^{\dagger}$, Andrea Mambretti*, Sajjad Arshad ${ }^{\ddagger}$, Michael Weissbacher*, \\ William Robertson*, Engin Kirda*, and Manuel Egele ${ }^{\dagger}$ \\ $\dagger$ Boston University \\ ${ }^{* \ddagger}$ Northeastern University \\ $\dagger\{$ wdblair, megele $\} @$ bu.edu, $*\{$ mbr, mw, wkr, ek $\} @$ ccs.neu.edu, ${ }^{\ddagger}$ arshad@iseclab.org
}

\begin{abstract}
Fifteen billion devices run Java and many of them are connected to the Internet. As this ecosystem continues to grow, it remains an important task to discover any unknown security threats these devices face. Fuzz testing repeatedly runs software on random inputs in order to trigger unexpected program behaviors, such as crashes or timeouts, and has historically revealed serious security vulnerabilities. Contemporary fuzz testing techniques focus on identifying memory corruption vulnerabilities that allow adversaries to achieve either remote code execution or information disclosure. Meanwhile, Algorithmic Complexity (AC) vulnerabilities, which are a common attack vector for denial-ofservice attacks, remain an understudied threat.
\end{abstract}

In this paper, we present HotFuzz, a framework for automatically discovering AC vulnerabilities in Java libraries. HotFuzz uses micro-fuzzing, a genetic algorithm that evolves arbitrary Java objects in order to trigger the worst-case performance for a method under test. We define Small Recursive Instantiation (SRI) as a technique to derive seed inputs represented as Java objects to micro-fuzzing. After micro-fuzzing, HotFuzz synthesizes test cases that triggered $\mathrm{AC}$ vulnerabilities into Java programs and monitors their execution in order to reproduce vulnerabilities outside the fuzzing framework. HotFuzz outputs those programs that exhibit high CPU utilization as witnesses for AC vulnerabilities in a Java library.

We evaluate HotFuzz over the Java Runtime Environment (JRE), the 100 most popular Java libraries on Maven, and challenges contained in the DARPA Space and Time Analysis for Cybersecurity (STAC) program. We evaluate SRI's effectiveness by comparing the performance of micro-fuzzing with SRI, measured by the number of $\mathrm{AC}$ vulnerabilities detected, to simply using empty values as seed inputs. In this evaluation, we verified known AC vulnerabilities, discovered previously unknown AC vulnerabilities that we responsibly reported to vendors, and received confirmation from both IBM and Oracle. Our results demonstrate that micro-fuzzing finds $\mathrm{AC}$ vulnerabilities in realworld software, and that micro-fuzzing with SRI-derived seed inputs outperforms using empty values.

\section{INTRODUCTION}

Software continues to be plagued by vulnerabilities that allow attackers to violate basic software security properties. These vulnerabilities take myriad forms, for instance failures to enforce memory safety that can lead to arbitrary code execution (integrity violations) or failures to prevent sensitive data from

Network and Distributed Systems Security (NDSS) Symposium 2020 23-26 February 2020, San Diego, CA, USA

ISBN 1-891562-61-4

https://dx.doi.org/10.14722/ndss.2020.24415

www.ndss-symposium.org being released to unauthorized principals (confidentiality violations). The third traditional security property, availability, is not exempt from this issue. However, denial-of-service (DoS) as a vulnerability class tends to be viewed as simplistic, noisy, and easy (in principle) to defend against.

This view, however, is simplistic, as availability vulnerabilities and exploits against them can take sophisticated forms. Algorithmic Complexity (AC) vulnerabilities are one such form, where a small adversarial input induces worst-case ${ }^{1}$ behavior in the processing of that input, resulting in a denial of service.

While in the textbook example against a hash table an adversary inserts values with colliding keys to degrade the complexity of lookup operations from an expected $O(1)$ to $O(n)$, the category of AC vulnerabilities is by no means hypothetical. Recent examples of algorithmic complexity vulnerabilities include denial of service issues in Go's elliptic curve cryptography implementation [4], an $\mathrm{AC}$ vulnerability that manifests through amplification of API requests against Netflix' internal infrastructure triggered by external requests [14], and a denial of service vulnerability in the Linux kernel's handling of TCP packets [3]. The vulnerability in the Linux kernel was considered serious enough that it was embargoed until OS vendors and large Linux users such as cloud providers and content delivery networks could develop and deploy patches. While these particular vulnerabilities involved unintended CPU time complexity, $\mathrm{AC}$ vulnerabilities can also manifest in the spatial domain for resources such as memory, storage, or network bandwidth.

While discovering AC vulnerabilities is notoriously challenging, program analysis seems like a natural basis for developing solutions to tackle such issues. In fact, prior research has started to explore program analysis techniques for finding $\mathrm{AC}$ vulnerabilities in software. Most of this work is based on manual or static analysis that scales to real world code bases, but focuses on detecting known sources of AC vulnerabilities, such as triggering worst case performance of commonly used data structures [19], regular expression engines [32], [57], [62], or serialization APIs [21].

Fuzz testing, where a fuzzer feeds random input to a program under test until the program either crashes or times out, has historically revealed serious bugs that permit Remote CodeExecution (RCE) exploits in widely used software such as operating system kernels, mobile devices, and web browsers.

\footnotetext{
${ }^{1}$ Strictly speaking, it is sufficient for attacks to cause bad behavior, it need not be "worst-case".
} 
Recent work has adapted existing state-of-the-art fuzz testers such as AFL [64] and libFuzzer [7] to automatically slow down programs with known performance problems. These approaches include favoring inputs that maximize the length of an input's execution in a program's Control Flow Graph (CFG) [47], incorporating multi-dimensional feedback that provides AFL with more visibility into the portions of the CFG each test case executes the most [37], and augmenting AFL with symbolic execution to maximize a Java program's resource consumption [43]. These recent advances demonstrate that modern fuzzers can automatically slow down programs such as sorting routines, hash table operations, and common Unix utilities.

These recent developments present exciting new directions for fuzz testing beyond detecting memory corruption bugs. However, these approaches do not reconcile the traditional fuzzing objective function of maximizing code coverage (breadth) with the opposing goal of maximizing a given program or individual method's runtime (depth). Indeed, these tools are evaluated by the slowdown they can achieve for a given program, as opposed to the amount of code they successfully cover. Achieving high code coverage on any program under test is a notoriously difficult task because common program patterns like comparing input to magic values or checksum tests are difficult to bypass using fuzzing alone, although program transformation tricks like splitting each comparison into a series of one byte comparisons [36] or simply removing them from the program [46] can improve coverage. Augmenting fuzzing with advanced techniques like taint analysis [50] or symbolic execution [44], [58] helps overcome these fuzzing roadblocks, and RedQueen [12] showed how advanced tracing hardware can emulate these more heavyweight techniques by providing a fuzzer with enough information to establish correspondence between program inputs and internal program state. Prior work has successfully shown fuzz testing can reproduce known AC vulnerabilities in software, and research continues to produce innovative ways to maximize code coverage. What is missing in fuzzing for $\mathrm{AC}$ vulnerabilities are techniques to automatically sanitize a program's execution for AC vulnerabilities, analogous to how modern fuzzers rely on sanitizers to detect memory corruption bugs [54]. Current fuzzing approaches in general lack the ability to automatically fuzz programs at the method level without the need for manually defined test harnesses.

This paper proposes micro-fuzzing (a concept analogous to micro-execution [24]) as a novel technique to automatically construct test harnesses that allow a fuzzer to invoke methods and sanitize their execution for AC vulnerabilities. Both AFL and libFuzzer can fuzz individual methods, but only after an analyst manually defines a test harness that transforms a flat bitmap into the types required to call a method. For AFL this involves defining a $\mathrm{C}$ program that reads the bitmap from standard input, whereas libFuzzer passes the bitmap to a specific function that it expects will call the method under test with the appropriate types derived from the bitmap.

In contrast, micro-fuzzing takes whole programs or libraries as input and attempts to automatically construct a test harness for every function contained in the input. Observe that this approach is analogous to micro-execution [24], which executes arbitrary machine code by using a virtual machine as a test harness that provides state on-demand in order to run the code under test. To this end, micro-fuzzing constructs test harnesses represented as function inputs, directly invokes functions on those inputs, and measures the amount of resources each input consumes using model specific registers available on the host machine. This alleviates the need to define test harnesses manually, and supports fuzzing whole programs and libraries by considering every function within them as a possible entrypoint. Furthermore, we sanitize every function's execution so that once its observed runtime crosses a configured threshold, we kill the micro-fuzzing process and highlight the function as vulnerable. This sanitization highlights functions with potential $\mathrm{AC}$ vulnerabilities out of all the functions micro-fuzzing automatically executes, as opposed to measuring a fuzzer's ability to automatically slow-down individual programs or functions.

We implement micro-fuzzing for Java programs in HotFuzz, which uses a genetic algorithm to evolve method inputs with the goal to maximize method execution time. Java provides an ideal platform for evaluating micro-fuzzing because of its wide use across different domains in industry and the JVM's support for introspection allows HotFuzz to automatically generate test harnesses, represented as valid Java objects, for individual methods dynamically at runtime. To generate initial populations of inputs, we devise two different strategies. The Identity Value Instantiation (IVI) strategy creates inputs by assigning each actual parameter the identity element of the parameter's domain (e.g., 0 for numeric types or "” for strings). In contrast, Small Recursive Instantiation (SRI) assigns parameters small values chosen at random from the parameter's domain. We use IVI for the sole purpose of providing a baseline for measuring the effectiveness of using SRI to generate seed inputs for micro-fuzzing, based on recent recommendations for evaluating new fuzz testing techniques [33].

Irrespective of how inputs are instantiated, HotFuzz leverages the EyeVM, an instrumented JVM that provides run-time measurements at method-level granularity. If micro-fuzzing creates an input that causes the method under test's execution time to exceed a threshold, HotFuzz marks the method as potentially vulnerable to an AC attack. To validate potential AC vulnerabilities, HotFuzz synthesizes Java programs that invoke flagged methods on the suspect inputs and monitors their endto-end execution in an unmodified JVM that mirrors a production environment. Those programs that exceed a timeout are included in HotFuzz's output corpus. Every program contained in the output corpus represents a witness of a potential AC vulnerability in the library under test that a human operator can either confirm or reject. Sanitizing method execution for AC vulnerabilities based on a threshold mimics the sanitizers used by modern fuzzers that kill a process whenever an integrity violation occurs at runtime, but it also introduces false positives into our results given that it is difficult to configure a proper timeout that detects only true positives. In our evaluation, we show that the number of bugs detected by our sanitizer is concise enough to permit manual analysis of the results.

We evaluate HotFuzz by micro-fuzzing the Java Runtime Environment (JRE), challenges provided by the DARPA Space and Time Analysis for Cybersecurity (STAC) program, and the 100 most popular libraries available on Maven, a popular repository for hosting Java program dependencies. We identify 5 intentional (in STAC) and 158 unintentional (in the JRE and 
Maven libraries) AC vulnerabilities.

In summary, this paper makes the following contributions:

- We introduce micro-fuzzing as a novel and efficient technique for identifying AC vulnerabilities in Java programs (see Section III-A).

- We devise two strategies (IVI and SRI) to generate seed inputs for micro-fuzzing (see Section III-A2b).

- We propose the combination of IVI and SRI with microfuzzing to detect AC vulnerabilities in Java programs.

- We design and evaluate HotFuzz, an implementation of our micro-fuzzing approach, on the Java Runtime Environment (JRE), challenges developed during the DARPA STAC program, and the 100 most popular libraries available on Maven. Our evaluation results yield previously unknown $\mathrm{AC}$ vulnerabilities in real-world software, including 26 in the JRE, 132 across 47 Maven libraries, including the widely used org.json library, "solve" 5 challenges from the STAC program, and include confirmations from IBM and Oracle. In addition, micro-fuzzing with SRI-derived seed inputs outperforms IVI-derived seed inputs, measured by the number of AC witnesses detected (see Section V).

\section{BACKGROUND AND THREAT MODEL}

In this section, we briefly describe Algorithmic Complexity (AC) vulnerabilities, different approaches that detect such vulnerabilities, the threat model we assume, and the high-level design goals of this work.

\section{A. AC Vulnerabilities}

AC vulnerabilities arise in programs whenever an adversary can provide inputs that cause the program to exceed desired (or required) bounds in either the spatial or temporal domains. One can define an AC vulnerability in terms of asymptotic complexity (e.g., an input of size $n$ causes a method to store $O\left(n^{3}\right)$ bytes to the filesystem instead of the expected $O(n)$ ), in terms of a concrete function of the input (e.g., an input of size $n$ causes a method to exceed the intended maximum $150 n$ seconds of wall clock execution time), or in other more qualitative senses (e.g., "the program hangs for several minutes"). However, in each case there is a definition, explicit or otherwise, of what constitutes an acceptable resource consumption threshold.

In this work, we assume an explicit definition of this threshold independent of a given program under analysis and rely on domain knowledge and manual filtering of $\mathrm{AC}$ witnesses in order to label those that should be considered as true vulnerabilities. We believe that this is a realistic assumption and pragmatic method for vulnerability identification that avoids pitfalls resulting from attempting to automatically understand intended resource consumption bounds, or from focusing exclusively on asymptotic complexity when in practice, as the old adage goes, "constants matter." We define an AC witness to be any input that causes a specific method under test's resource consumption to exceed a configured threshold. We consider any method that has an $\mathrm{AC}$ witness to contain an $\mathrm{AC}$ vulnerability.

We recognize that this definition of an AC vulnerability based on observing a method's resource consumption exceeding some threshold will inevitably cause some false positives, since the chosen threshold may not be appropriate for a given method under test. Section III presents a strategy for minimizing false positives by automatically reproducing AC vulnerabilities in a production environment outside our fuzzing framework. This step may fail to remove all false positives, and in our evaluation given in Section $\mathrm{V}$ we show that the output of this validation stage is concise enough to allow an analyst to manually triage the results. Since we make no assumption about the methods we test in our analysis, we believe observing output that consists of less than three hundred test cases is reasonable for a human analyst.

\section{B. AC Detection}

Software vulnerability detection in general can be roughly categorized as a static analysis, dynamic testing, or some combination of the two. Static analysis has been proposed to analyze a given piece of code for its worst case execution time behavior. While finding an upper bound to program execution time is certainly valuable, conservative approximations in static analysis systems commonly result in a high number of false positives. Furthermore, even manual interpretation of static analysis results in this domain can be challenging as it is often unclear whether a large worst-case execution time results from a property of the code or rather the approximation in the analysis. Additionally, static analyses for timing analysis commonly work best for well structured code that is written with such analysis in mind (e.g., code in a real-time operating system). The real-world generic code bases in our focus (e.g., the Java Runtime Environment), have not been engineered with such a focus and quickly reach the scalability limits of static timing analyzers.

Dynamic testing, in particular fuzz testing, has emerged as a particularly effective vulnerability detection approach that runs continuously in parallel with the software development lifecycle [41], [56]. State of the art fuzzers detect bugs by automatically executing a program under test instrumented with sanitizers until the program either crashes or times out. A sanitized program crashes immediately after it violates an invariant enforced by the sanitizer, such as writing past the boundary of a buffer located on the stack or reading from previously freed memory. Once a fuzzer generates a test case that crashes a given sanitized program under test, the test case is a witness for a memory corruption bug in the original program. Since memory corruption bugs may be extended into exploits that achieve Remote Code Execution or Information Disclosure, fuzzers offer an effective and automated approach to software vulnerability detection. When source code is not available, a fuzzer can still attempt to crash the program under test in either an emulated or virtualized environment.

Fuzz testing's utility for detecting memory corruption bugs in programs is well known, and current research explores how to maximize both the amount of code a fuzzer can execute and the number of bugs a fuzzer can find. Unfortunately, defining a sanitizer that crashes a process after an $\mathrm{AC}$ vulnerability occurs is not as straightforward as detecting memory integrity violations. This is in part because what constitutes an AC vulnerability heavily depends on the program's domain. For example, a test case that slows down a program by several milliseconds may be considered an $\mathrm{AC}$ vulnerability for a low latency financial trading application and benign for a web service that processes requests asynchronously. 
In this work, we propose a sanitizer in HotFuzz that kills a process after a method's runtime exceeds a configured threshold. Like sanitizers for memory corruption bugs, this allows us to save only those test cases that exhibit problematic behavior. The drawback is that we do not have absolute certainty that our test cases are actual bugs in the original program and risk highlighting test cases as false positives. Building a fuzzing analysis that does not introduce any false positives is notoriously difficult, and fuzzers that detect memory corruption bugs are not immune to this problem. For example, Aschermann et al. [12] point out that previous evaluations erroneously report crashing inputs that exhaust the fuzzer's available memory as bugs in the original program under test. Furthermore, sanitizers point out many different sources of bugs including stack based overflows, use after free, use after return, and heap based overflows. While the presence of any of these bugs is problematic, triaging is still required to understand the problem given in a test case.

\section{Fuzzing AC}

SlowFuzz [47] and PerfFuzz [37], adapt two state of the art fuzzers, libFuzzer and AFL, respectively, and demonstrate the capability to automatically slow down individual programs or methods implemented in $\mathrm{C} / \mathrm{C}++$. Parallel developments also showed frameworks built on top of AFL can successfully slow down programs in interpreted languages as well [43].

HotFuzz departs from these previous works by automatically creating test harnesses during micro-fuzzing, and sanitizing method execution for AC vulnerabilities. In contrast to these tools, HotFuzz does not require an analyst to manually define a test harness in order to fuzz individual methods contained in a library. This key feature differentiates micro-fuzzing found in HotFuzz from how AFL or libFuzzer fuzz individual methods. Since AFL and LibFuzzer only consider test cases consisting of flat bitmaps, one can fuzz an individual method with AFL by defining a test harness that transforms a bitmap read from stdin into function inputs, and with libFuzzer an analyst implements a $\mathrm{C}$ function that takes the test case as input and must transform it into the types needed to invoke a function. Observe that this must be done manually, whereas HotFuzz examines the type signature of the method under test and attempts to generate the test harness automatically. To reproduce our evaluation using existing tools, we would need to manually define approximately 400,000 individual test harnesses for all the artifacts contained in our evaluation.

SlowFuzz and PerfFuzz both explore how fuzzers can automatically slow down individual programs. Understanding what techniques work best to slow down code is necessary to understand how to design a fuzzer to detect AC vulnerabilities. SlowFuzz observed that using the number of executed instructions as a test case's fitness in libFuzzer's genetic algorithm can be used to slow down code with known performance problems, such as sorting routines and hash table implementations. PerfFuzz went a step further and showed how incorporating a performance map that tracks the most visited edges in a program's CFG can help a fuzzer further slow down programs.

These approaches take important steps needed to understand what techniques allow fuzzers to automatically slow down arbitrary code in order to spot AC vulnerabilities in programs. At the same time, they lack three important properties for being used to detect unknown $\mathrm{AC}$ vulnerabilities. First, they require manually defined test harnesses in order to fuzz individual functions. Second, these fuzzing engines only consider flat bitmaps as input to the programs under test, and miss the opportunity to evolve the high level classes of the function's domain in the fuzzer's genetic algorithm. Finally, these tools are meant to understand what techniques successfully slow down code the most, and do not provide a method for sanitizing method execution for $\mathrm{AC}$ vulnerabilities and presenting these results to a human analyst.

\section{Optimization}

The goal of identifying $\mathrm{AC}$ vulnerabilities boils down to a simple to posit yet challenging to answer optimization question. "What are concrete input values that make a given method under test consume the most resources?" One possible approach to tackle such optimization problems is with the help of genetic algorithms. A genetic algorithm emulates the process of evolution to derive approximations for a given optimization problem. To this end, a genetic algorithm will start with an initial population of individuals and over the duration of multiple generations repeatedly perform three essential steps: i) Mutation, ii) Crossover, and iii) Selection. In each generation, a small number of individuals in the population may undergo mutation. Furthermore, each generation will see a large number of crossover events where two individuals combine to form offspring. Finally, individuals in the resulting population get evaluated for their fitness, and the individuals with the highest fitness are selected to form the population for the next generation. The algorithm stops after either a fixed number of generations, or when the overall fitness of subsequent populations no longer improves. In our scenario where we seek to identify AC vulnerabilities in Java methods, individuals correspond to the actual parameter values that are passed to a method under test. Furthermore, assessing fitness of a given individual can be accomplished by measuring the method's resource consumption while processing the individual (see Section IV-A). While mutation and crossover are straightforward to define on populations whose individuals can be represented as sequences of binary data, the individuals in our setting are tuples of well-formed Java objects. As such, mutation and crossover operators must work on arbitrary Java classes, as opposed to flat binary data (see Section III-A1).

\section{E. Threat Model}

In this work, we assume the following adversarial capabilities. An attacker either has access to the source code of a targeted program and its dependencies, or a compiled artifact that can be tested offline. Using this code, the attacker can employ arbitrary techniques to discover AC vulnerabilities exposed by the program, either in the program itself or by any library functionality invoked by the program. Furthermore, we assume that these vulnerabilities can be triggered by untrusted input.

An adversary can achieve DoS attacks on programs and services that utilize vulnerable libraries by taking the information they learn about a library through offline testing and developing exploits that trigger the $\mathrm{AC}$ vulnerabilities contained in library methods used by a victim program. For example, an adversary could take the test cases produced by our evaluation (see Section V) and attempt to reproduce their behavior on programs that utilize the methods. Determining whether an 
adversary can transform these test cases into working AC exploits on victim programs is outside the scope of this work.

\section{F. Design Goals}

The goal of our work is to discover $\mathrm{AC}$ vulnerabilities in Java code so that they can be patched before attackers have the opportunity to exploit them. In particular, we aim for an analysis that is automated and efficient such that it can run continuously in parallel with the software development lifecycle on production artifacts. This gives developers insight into potential vulnerabilities hiding in their applications without altering their development workflow.

\section{HOTFuZZ OVERVIEW}

HotFuzz adopts a dynamic testing approach to detecting $\mathrm{AC}$ vulnerabilities, where the testing procedure consists of two phases: ( $i$ ) micro-fuzzing, and (ii) witness synthesis and validation. In the first phase, a Java library under test is submitted for micro-fuzzing, a novel approach to scale AC vulnerability detection. In this process, the library is decomposed into individual methods, where each method is considered a distinct entrypoint for testing by a $\mu F u z z$ instance. As opposed to traditional fuzzing, where the goal is to provide inputs that crash a program under test, here each $\mu$ Fuzz instance attempts to maximize the resource consumption of individual methods under test using genetic optimization over the method's inputs. To that end, seed inputs for each method under test are generated using one of two instantiation strategies: Identity Value Instantiation (IVI) and Small Recursive Instantiation (SRI). Method-level resource consumption when executed on these inputs is measured using a specially-instrumented Java virtual machine we call the EyeVM. If optimization eventually produces an execution that is measured to exceed a pre-defined threshold, then that test case is forwarded to the second phase of the testing procedure.

Differences between the micro-fuzzing and realistic execution environments can lead to false positives. The purpose of the second phase is to validate whether test cases found during micro-fuzzing represent actual vulnerabilities when executed in a real Java run-time environment, and therefore reduce the number of false positives in our final results. This validation is achieved through witness synthesis where, for each test case discovered by the first phase, a program is generated that invokes the method under test with the associated inputs that produce abnormal resource usage. If the behavior with respect to resource utilization that was observed during micro-fuzzing is replicated, then the synthesized test case is flagged as a witness of the vulnerability that can then be examined by a human analyst. Otherwise, we discard the synthesized test case as a false positive.

Figure 1 depicts a graphical overview of the two phases. In the following, we motivate and describe the design of each component of the testing procedure in detail.

\section{A. Micro-Fuzzing}

Micro-fuzzing represents a drastically different approach to vulnerability detection than traditional automated wholeprogram fuzzing. In the latter case, inputs are generated for an entire program either randomly, through mutation of seed inputs, or incorporating feedback from introspection on execution. Whole-program fuzzing has the significant benefit that any abnormal behavior-i.e., crashes-that is observed should be considered as a real bug as by definition all the constraints on the execution path that terminates in the bug are satisfied (up to the determinism of the execution). However, whole-program fuzzing also has the well-known drawback that full coverage of the test artifact is difficult to achieve. Thus, an important measure of a traditional fuzzer's efficacy is its ability to efficiently cover paths in a test artifact.

Micro-fuzzing strikes a different trade-off between coverage and path satisfiability. Inspired by the concept of microexecution [24], micro-fuzzing constructs realistic intermediate program states, defined as Java objects, and directly executes individual methods on these states. Thus, we can cover all methods by simply enumerating all the methods that comprise a test artifact, while the difficulty lies instead in ensuring that constructed states used as method inputs are feasible in practice. $^{2}$ In our problem setting, where we aim to preemptively warn developers against insecure usage of AC-vulnerable methods or conservatively defend against powerful adversaries, we believe micro-fuzzing represents an interesting and useful point in the design space that complements whole program fuzzing approaches. In this work, we consider the program's state as the inputs given to the methods we micro-fuzz. Modeling implicit parameters, such as files, static variables, or environment variables are outside the scope of this work.

A second major departure from traditional fuzzing is the criteria used to identify vulnerabilities. Typical fuzzers use abnormal termination as a signal that a vulnerability might have been found. In our case, vulnerabilities are represented not by crashes but rather by excessive resource consumption. Thus, coverage is not the sole metric that must be maximized in our case. Instead, HotFuzz must balance between maximizing a method's resource utilization in addition to coverage. Conceptually speaking, implementing resource measurement is a straightforward matter of adding methods to the existing Reflection API in Java that toggles resource usage recording and associates measurements with Java methods. In practice, this involves non-trivial engineering, the details of which we present in Section IV. In the following, we describe how HotFuzz optimizes resource consumption during microfuzzing given dynamic measurements provided by the EyeVM, our instrumented JVM that provides run-time measurements at method-level granularity.

1) Resource Consumption Optimization: HotFuzz's fuzzing component, called $\mu \mathrm{Fuzz}$, is responsible for optimizing the resource consumption of methods under test. To do so, $\mu \mathrm{Fuzz}$ uses genetic optimization to evolve an initial set of seed inputs over multiple generations until it detects abnormal resource consumption. Traditional fuzzers use evolutionary algorithms extensively, but in this work we present a genetic optimization approach to fuzzing that departs from prior work in two important ways. First, as already discussed, traditional fuzzers optimize an objective function that solely considers path coverage (or some proxy thereof), whereas in our setting we are concerned in addition with resource consumption. Prior work for detecting $\mathrm{AC}$ vulnerabilities through fuzz testing either record resource consumption using a combination of program instrumentation, CPU utilization, or counting executed instructions. In contrast, we record resource consumption

\footnotetext{
${ }^{2}$ We note that in the traditional fuzzing case, a similar problem exists in that while crashes indicate the presence of an availability vulnerability, they do not necessarily represent exploitable opportunities for control-flow hijacking.
} 


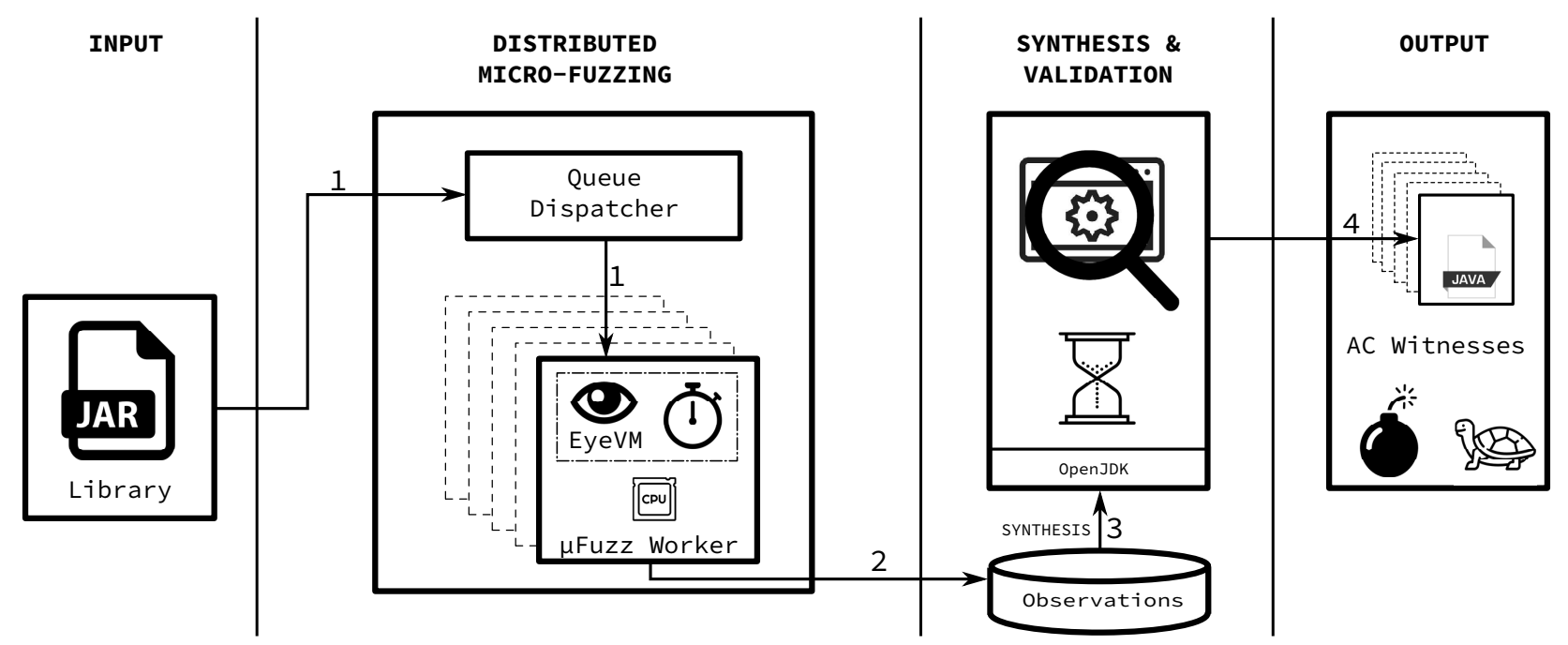

Fig. 1: Architectural overview of the HotFuzz testing procedure. In the first phase, individual $\mu$ Fuzz instances micro-fuzz each method comprising a library under test. Resource consumption is maximized using genetic optimization over inputs seeded using either IVI or SRI. In the second phase, test cases flagged as potential AC vulnerabilities by the first phase are synthesized into Java programs. These programs are executed in an unmodified JVM in order to replicate the abnormal resource consumption observed in the first phase. Programs that fail to do so are rejected as false positives. HotFuzz reports those programs that pass the Synthesis and Validation stage as AC vulnerability witnesses to a human analyst.

using an altered execution environment (the EyeVM) and require no modification to the library under test. Second, traditional fuzzers treat inputs as flat bitmaps when genetic optimization (as opposed to more general mutation) is applied. Recall that genetic algorithms require defining crossover and mutation operators on members of the population of inputs. New generations are created by performing crossover between members in prior generations. Additionally, in each generation, some random subset of the population undergoes mutation with small probability. Since $\mu$ Fuzz operates on Java values rather than flat bitmaps, we must define new crossover and mutation operators specific to this domain as bitmap-specific operators do not directly translate to arbitrary Java values, which can belong to arbitrary Java classes.

a) Java Value Crossover: Genetic algorithms create new members of a population by "crossing" existing members. When individual inputs are represented as bitmaps, a standard approach is single-point crossover: a single offset into two bitmaps is selected at random, and two new bitmaps are produced by exchanging the content to the right of the offset from both parents. Single-point crossover does not directly apply to inputs comprised of Java objects, but can be adapted in the following way. Let $X_{0}, X_{1}$ represent two existing inputs from the overall population and $\left(x_{0}, x_{1}\right)_{0}=x_{0}$ and $\left(x_{0}, x_{1}\right)_{1}=x_{1}$. To produce two new inputs, perform single-point crossover for each corresponding pair of values $\left(x_{0}, x_{1}\right) \in\left(X_{0}, X_{1}\right)$ using

$$
\left(x_{0}^{\prime}, x_{1}^{\prime}\right)= \begin{cases}C\left(x_{0}, x_{1}\right) & \text { if }\left(x_{0}, x_{1}\right) \text { are primitives } \\ \left(C_{L}\left(x_{0}, x_{1}\right), C_{R}\left(x_{0}, x_{1}\right)\right) & \text { if }\left(x_{0}, x_{1}\right) \text { are objects. }\end{cases}
$$

Here, $C$ performs one-point crossover directly on primitive values and produces the offspring as a pair. When $x_{0}$ and $x_{1}$ are objects, $C_{L}$ and $C_{R}$ recursively perform cross-over on every member attribute in $\left(x_{0}, x_{1}\right)$ and select the left and right offspring, respectively. For example, consider a simple Java class List that implements a singly linked list. The
List class consists of an integer attribute hd and a List attribute $t l$. Crossing an instance of List $\vec{x}$ with another instance $\vec{y}$ constructs two new lists $\overrightarrow{x^{\prime}}$ and $\overrightarrow{y^{\prime}}$ given by

$$
\begin{aligned}
& \overrightarrow{x^{\prime}}=C_{L}(\vec{x}, \vec{y})=\left(h d:=C(\vec{x} \cdot h d, \vec{y} \cdot h d)_{0}, t l:=C_{L}(\vec{x} . t l, \vec{y} . t l)\right) \\
& \overrightarrow{y^{\prime}}=C_{R}(\vec{x}, \vec{y})=\left(h d:=C(\vec{x} \cdot h d, \vec{y} \cdot h d)_{1}, t l:=C_{R}(\vec{x} . t l, \vec{y} \cdot t l)\right)
\end{aligned}
$$

In this example we show how HotFuzz crosses over a List that holds Integers, but if the type of value stored in the hd attribute were a complex class $T$, the crossover operator would recursively apply crossover to every attribute stored in $T$.

b) Java Value Mutation: Mutation operators for traditional fuzzers rely on heuristics to derive new generations, mutating members of the existing population through random or semicontrolled bit flips. In contrast, micro-fuzzing requires mutating arbitrary Java values, and thus bitmap-specific techniques do not directly apply.

Instead, $\mu$ Fuzz mutates Java objects using the following procedure. For a given Java object $x$ with attributes $\left\{a_{0}, a_{1}, \ldots, a_{n}\right\}$, choose one of its attributes $a_{i}$ uniformly at random. Then we define the mutation operator $M$ as

$$
a_{i}^{\prime}= \begin{cases}M_{\text {flip_bit }}\left(a_{i}\right) & \text { if } a_{i} \text { is a numeric value, } \\
M_{\text {insert_char }}\left(a_{i}\right) & \text { if } a_{i} \text { is a string or array value, } \\
M_{\text {delete_char }}\left(a_{i}\right) & \text { if } a_{i} \text { is a string or array value, } \\
M_{\text {replace_char }}\left(a_{i}\right) & \text { if } a_{i} \text { is a string or array value, } \\
M_{\text {swap_chars }}\left(a_{i}\right) & \text { if } a_{i} \text { is a string or array value, } \\
M_{\text {mutate_attr }}\left(a_{i}\right) & \text { if } a_{i} \text { is an object. }\end{cases}
$$

Each mutation sub-operator above operates on the attribute $a_{i}$ chosen from the object $x$. For example, $M_{\text {flip_bit }}$ selects a bit at random in a numeric element and flips it, while $M_{\text {swap_chars }}$ randomly selects two elements of a string or array and swaps 
them. In our current implementation, we only consider arrays of primitive types. The other sub-operators are defined in an intuitively similar manner.

When an attribute is a class, as opposed to a primitive type or a string or array, mutation utilizes the $M_{\text {mutate_attr }}$ operator. $M_{\text {mutate_attr }}$ recursively applies the mutation operator $M$ to the chosen attribute $a_{i}$ when $a_{i}$ is an object. After we obtain the mutated attribute $a_{i}^{\prime}$, we produce the mutated object $x^{\prime}$ by replacing $a_{i}$ with $a_{i}^{\prime}$ in $x$.

2) Seed Generation: Given suitable crossover and mutation operators, all that remains to apply standard genetic optimization is the definition of a procedure to generate seed inputs. We define two such procedures that we describe below: Identity Value Instantiation (IVI), and Small Recursive Instantiation (SRI).

a) Identity Value Instantiation: Recent work has proposed guidelines for evaluating new fuzz testing techniques [33]. One of these guidelines is to compare any proposed strategy for constructing seed inputs for fuzz testing with "empty" seed inputs. Intuitively, empty seed inputs represent the simplest possible seed selection strategy. Since empty bitmaps do not directly translate to our input domain, we define IVI as an equivalent strategy for Java values. The term "identity value" is derived from the definition of an identity element for an additive group.

In particular, IVI is defined as

$$
I(T)= \begin{cases}0 & \text { if } T \text { is a numeric type, } \\
\text { false } & \text { if } T \text { is a boolean, } \\
\{\} & \text { if } T \text { is a string, } \\
T_{\text {random }}\left(I\left(T_{0}\right), \ldots, I\left(T_{n}\right)\right) & \text { if } T \text { is an array, } \\
\text { if } T \text { is a class. }\end{cases}
$$

That is, $I(T)$ selects the identity element for all primitive types, while for classes $I$ is recursively applied to all parameter types $T_{i}$ of a randomly selected constructor for $T$. Thus, for a given method under test $M, I(M)$ is defined as $I$ applied to each of $M$ 's parameter types.

b) Small Recursive Instantiation: In addition to IVI, we define a complementary seed input generation procedure called Small Recursive Instantiation (SRI). In contrast to IVI, SRI generates random values for each method parameter. However, experience dictates that selecting uniformly random values from the entire range of possible values for a given type is not the most productive approach to input generation. For example, starting with large random numbers as seed inputs may waste time executing benign methods that simply allocate large empty data structures like Lists or Sets. For example, creating a List with the Arraylist (int capacity) constructor and passing it an initial capacity of $1<<30$ takes over 1 second and requires over $4 \mathrm{~GB}$ of RAM. For this reason, we configure SRI with a spread parameter $\alpha$ that limits the range of values from which SRI will sample. Thus, SRI is defined as

$$
S(T, \alpha)= \begin{cases}R_{\text {num }}(-\alpha, \alpha) & \text { if } T \text { is a numeric type } \\
\left\{R_{\text {char }}\right\}_{\text {num }}(0, \alpha) & \text { if } T \text { is a string, } \\
\{S(T, \alpha)\}_{\text {num }}(0, \alpha) & \text { if } T \text { is an array, } \\
T_{\text {random }}\left(S\left(T_{0}, \alpha\right), \ldots, S\left(T_{n}, \alpha\right)\right) & \text { if } T \text { is a class. }\end{cases}
$$

In the above, $R_{\text {num }}(x, y)$ selects a value at random from the range $[x, y)$, while $R_{\text {char }}$ produces a character obtained at random. Similarly to $I$, for a given method under test $M$ we define $S(M)$ as $S$ applied to each of $M$ 's parameter types. We note that SRI with $\alpha=0$ is in fact equivalent to IVI, and thus IVI can be considered a special case of SRI. We note that HotFuzz can be configured with different random number distributions to alter the behavior of $R$.

\section{B. Witness Synthesis}

Test cases exhibiting abnormal resource consumption are forwarded from the micro-fuzzing phase of the testing procedure to the second phase: witness synthesis and validation. The rationale behind this phase is to reproduce the behavior during fuzzing in a realistic execution environment using a real JVM in order to avoid false positives introduced due to the measurement instrumentation.

In principle, one could simply interpret any execution that exceeds the configured timeout as evidence of a vulnerability. In practice, this is an insufficient criterion since the method under test could simply be blocked on I/O, sleeping, or performing some other benign activity. An additional consideration is that because the EyeVM operates in interpreted mode during the first micro-fuzzing stage (see Section IV-B), a test case that exceeds the timeout in the first phase might not do so during validation when JIT is enabled.

Therefore, validation of suspected vulnerabilities in a realistic environment is necessary. To that end, given an abnormal method invocation $M\left(v_{0}, \ldots, v_{n}\right)$, a self-contained Java program is synthesized that invokes $M$ by using a combination of the Reflection API and the Google GSON library. The program is packaged with any necessary library dependencies and is then executed in a standard JVM with JIT enabled. Instead of using JVM instrumentation, the wall clock execution time of the entire program is measured. If the execution was both CPU-bound as measured by the operating system and the elapsed wall clock time exceeds a configured timeout, the synthesized program is considered a witness for a legitimate $\mathrm{AC}$ vulnerability and recorded in serialized form in a database. The resulting corpus of AC vulnerability witnesses are reported to a human analyst for manual examination.

Recall HotFuzz takes compiled whole programs and libraries as input. Therefore, the witnesses contained in its final output corpus do not point out the root cause of any vulnerabilities in a program's source code. However, the EyeVM can trace the execution of any Java program running on it (see Section IV-A2). Given a witness of an AC vulnerability, we can trace its execution in the EyeVM in order to gain insight into the underlying causes of the problem in the program or library. In Section V, we use this technique to discover the root cause for several AC bugs detected by HotFuzz.

\section{IMPLEMENTATION}

In this section, we describe our prototype implementation of HotFuzz and discuss the relevant design decisions. Our prototype implementation consists of 5,487 lines of Java code, 1,007 lines of $\mathrm{C}++$ code in the JVM, and 288 lines of Python code for validating $\mathrm{AC}$ witnesses detected by micro-fuzzing.

\section{A. EyeVM}

The OpenJDK includes the HotSpot VM, an implementation of the Java Virtual Machine (JVM), and the libraries and toolchain that support the development and execution of Java programs. The EyeVM is a fork of the OpenJDK that includes a modified HotSpot VM for recording resource measurements. 
By modifying the HotSpot VM directly, our micro-fuzzing procedure is compatible with any program or library that runs on the OpenJDK.

The EyeVM exposes its resource usage measurement capabilities to analysis tools using the Java Native Interface (JNI) framework. In particular, a fuzzer running on the EyeVM can obtain the execution time of a given method under test by invoking the getRuntime() method which we added to the existing Executable class in the OpenJDK. The Executable class allows $\mu$ Fuzz to obtain a Java object that represents the method under test and access analysis data through our API. This API includes three methods to control and record our analysis: setMethodUnderTest, clearAnalysis, and get Runtime.

We chose to instrument the JVM directly because it allows us to analyze programs without altering them through bytecode instrumentation. This enables us to micro-fuzz a library without modifying it in any way. It also limits the amount of overhead introduced by recording resource measurements.

The EyeVM can operate in two distinct modes to support our resource consumption analysis: measurement, described in Section IV-A1, and tracing, described in Section IV-A2. In measurement mode, the EyeVM records program execution time with method-level granularity, while tracing mode records method-level execution traces of programs running on the EyeVM. HotFuzz utilizes the measurement mode to record the method under test's execution time during micro-fuzzing, while the tracing mode allows for manual analysis of the suspected AC vulnerabilities produced by HotFuzz.

1) EyeVM Measurement Mode: Commodity JVMs do not provide a convenient mechanism for recording method execution times. Prior work has made use of bytecode rewriting [35] for this purpose. However, this approach requires modifying the test artifact, and produced non-trivial measurement perturbation in our testing. Alternatively, an external interface such as the Serviceability Agent [52] or JVM Tool Interface [11] could be used, but these approaches introduce costly overhead due to context switching every time the JVM invokes a method. Therefore, we chose to collect resource measurements by instrumenting the HotSpot VM directly.

The HotSpot VM interprets Java programs represented in a bytecode instruction set documented by the JVM Specification [10]. During start up, the HotSpot VM generates a Template Table and allocates a slot in this table for every instruction given in the JVM instruction set. Each slot contains a buffer of instructions in the host machine's instruction set architecture that interprets the slot's bytecode. The Template Interpreter inside the HotSpot VM interprets Java programs by fetching the Java instruction given at the Bytecode Pointer (BCP), finding the instruction's slot in the Template Interpreter's table, and jumping to that address in memory. The HotSpot VM interprets whole Java programs by performing this fetch, decode, execute procedure starting from the program's entrypoint which is given by a method called ma in in one of the program's classes. During execution the Template Interpreter also heavily relies on functionality provided by HotSpot's C++ runtime.

The HotSpot source code contains an Assembler API that allows JVM developers to author $\mathrm{C}++$ methods that, when executed, generate the native executable code required for each slot in the Template Interpreter. This allows a developer to implement the functionality for a given bytecode instruction, such as iadd, by writing a C++ method $m$. When the HotSpot VM starts up, it invokes $m$, and $m$ emits as output native code in the host machine's instruction set architecture that interprets the iadd bytecode. HotSpot saves this native code to the appropriate slot so it can use it later to interpret iadd bytecode instructions. The API available to developers who author these methods naturally resembles the host's instruction set architecture. One can think of this Assembler API as a C++ library that resembles an assembler like GNU as. For example, if the two arguments to an iadd instruction reside in memory, a developer can call methods on this API to load the values into registers, add them together, and store the result on the JVM's operand stack. We use this API to emit code that efficiently records methods' resource utilization for our analysis.

We instrument the JVM interpreter by augmenting relevant slots in the Template Interpreter using the same API that the JVM developers use to define the interpreter. To measure execution time, we modify method entry and exit to store the method's elapsed time, measured by the RDTSC ModelSpecific Register (MSR) available on the $\times 86$ architecture, into thread-local data structures that analysis tools can query after a method returns. We limit our current implementation to the $\times 86-64$ platform, but this technique can be applied to any architecture supported by the HotSpot VM. In addition, we could modify the Template Interpreter further to record additional resources, such as memory or disk consumption.

Unfortunately, instrumenting the JVM such that every method invocation and return records that method's execution time introduces significant overhead. That is, analyzing a single method also results in recording measurements for every method it invokes in turn. This is both unnecessary and adds noise to the results due to both the need to perform an additional measurement for each method as well as the adverse effects on the cache due to the presence of the corresponding measurement field. Thus, our implementation avoids this overhead by restricting instrumentation to a single method under test that $\mu$ Fuzz can change on demand.

In particular, $\mu$ Fuzz stores the current method under test inside thread-local data. During method entry and exit, the interpreter compares the current method to the thread's method under test. If these differ, the interpreter simply jumps over our instrumentation code. Therefore, any method call outside our analysis incurs at most one comparison and a short jump.

Every time the interpreter invokes a method, our instrumentation stores the latest value of rdtsc into an attribute $T_{\text {start }}$ in the calling thread and increments a depth counter $T_{\text {depth }}$. If the same method enters again in a recursive call, we increment $T_{\text {depth }}$. If the method under test calls another method, it simply skips over our analysis code. Each time the method under test returns, we decrement $T_{\text {depth }}$. If $T_{\text {depth }}$ is equal to zero, the EyeVM invokes rdtsc and the computed difference between the current value and $T_{\text {start }}$ is stored inside the calling thread. Observe that the measured execution time for the method under test consequently includes its own execution time and the execution time of all the methods it invokes. This result is stored inside the method under test's internal JVM data structure located in its class's constant pool. The Assembler API available in the JVM sources supports all the functionality needed to implement these measurements, including com- 
puting the offsets of $\mathrm{C}++$ attributes, manipulating machine registers, and storing values to memory.

Every time the JVM invokes a method, the Template Interpreter sets up a new stack frame for the method which the interpreter removes after the method returns. The code that implements this logic is defined using the same Assembler API that implements each JVM bytecode instruction. To record our resource measurements, we insert relevant code snippets into the Template Interpreter that run every time the EyeVM adds or removes a stack frame.

The java executable that runs every Java program begins by loading the HotSpot VM as a shared library into its process address space in order to run the JVM. Thus, EyeVM can export arbitrary symbols to expose a JNI interface to analysis tools implemented in Java. Currently, the EyeVM defines functions that allow a process to configure the method under test, poll the method's most recent execution time, and to clear the method's stored execution time. The EyeVM then simply uses the JNI to bind the methods we added to the Executable Java class to the native EyeVM functions that support our analysis.

2) EyeVM Tracing Mode: In addition to measuring method execution times, EyeVM allows an analyst to trace the execution of Java programs with method-level granularity. Tracing provides valuable insight into programs under test and is used herein to evaluate HotFuzz's ability to detect AC vulnerabilities (see Section V). Each event given in a trace represents either a method invocation or return. Invocation events carry all parameters passed to the method as input.

In principle, traces could be generated either by instrumenting the bytecode of the program under test, or through an external tool interface like the JVMTI. As both of these approaches introduce significant overhead, we (as for measurement mode) opt instead for JVM-based instrumentation. That is, modifying the JVM directly to trace program execution does not require any modification of the program under analysis and only requires knowledge of internal JVM data structures.

EyeVM's tracing mode is implemented by instrumenting the bytecode interpreter generated at run-time by the HotSpot VM. Recall that the JVM executes bytecode within a generated Template Interpreter in the host machine's instruction set architecture. In order to generate program traces that record all methods invoked by the program under test, stubs are added to the locations in the Template Interpreter that invoke and return from methods. We note that these are the same locations that are instrumented to implement measurement mode.

However, while performance overhead is an important factor, program execution tracing can nevertheless be effectively implemented in the $\mathrm{C}++$ run-time portion of the JVM as opposed to generating inline assembly as in the measurement case. Then, during interpreter generation, all that is added to the generated code are invocations of the $\mathrm{C}++$ tracing functions.

To trace a program under test, we define a trace recording point as when the program either invokes a method or returns from one. When a method under test reaches a trace recording point the JVM is executing in the generated Template Interpreter represented in x86-64 assembly. Directly calling a $\mathrm{C}++$ function in this state will lead to a JVM crash, as the machine layout of the bytecode interpreter differs from the Application Binary
Interface (ABI) expected by the $\mathrm{C}++$ run-time. Fortunately, the JVM provides a convenient mechanism to call methods defined in the $\mathrm{C}++$ run-time using the $\mathrm{Call}$ _VM method available in the Assembler API. The Call_VM method requires that parameters passed to the $\mathrm{C}++$ function are contained within general purpose registers. This facility is used to pass a pointer to the object that represents the method we wish to trace, a value that denotes whether the event represents an invocation or return, and a pointer to the parameters passed to the method under test. All of this information is accessible from the current interpreter frame when tracing an event. The JVM maintains an Operand Stack that holds inputs to methods and bytecode instructions. Internally, a special variable called the Top of the Stack State (ToSState) allows the JVM to check where the top of the Operand Stack is located. Before calling our C++ stub to trace an event, we push the current ToSState onto the machine stack. Next, we call our $\mathrm{C}++$ tracing function. After the tracing function returns, we pop the ToSState off the machine stack and restore it to its original value.

The trace event stub itself collects the name of every invoked method or constructor, and its parameters. The name of the method is obtained from the method object the JVM passes to the stub. The parameters passed to the method under test are collected by accessing the stub parameters in similar fashion. The JVM's SignatureIterator class allows the tracing function to iterate over the parameter types specified in the method under test's signature, and, therefore, ensures that tracing records the correct parameter types. For each parameter passed to a method, both its type and value are saved. Values of primitive types are represented as literals, whereas objects are represented by their internal ID in the JVM. Within the trace file, one can find the origin of a given ID from the object's constructor in the trace. All of this information is streamed to a trace file one event at a time.

\section{B. $\mu F u z z$}

Micro-fuzzing is implemented using a message broker and a collection of $\mu$ Fuzz instances. Each $\mu$ Fuzz instance runs inside the EyeVM in measurement mode, consumes methods as jobs from a queue, and micro-fuzzes each method within its own process. Over time, micro-fuzzing methods in the same process might introduce side-effects that prevent future jobs from succeeding. For example, a method that starts an applet could restrict the JVM's security policy and prevent $\mu$ Fuzz from performing benign operations required to fuzz future methods. This occurs because once a running VM restricts its security policy, it cannot be loosened. To prevent this and similar issues from affecting future micro-fuzzing jobs, we add the following probe to every $\mu$ Fuzz instance. Prior to fuzzing each method received from the job queue, $\mu$ Fuzz probes the environment to ensure basic operations are allowed. If this probing results in a security exception, the $\mu$ Fuzz process is killed and a new one is spawned in its place. Traditional fuzzers avoid these problems by forking before each test case so it can run in a fresh state. For a simple Java program that loops indefinitely, the JVM runs 16 operating system threads. Constantly forking such a heavily multi-threaded environment on every test case introduces unnecessary complexity and very quickly destabilizes our experiments.

We configure each $\mu$ Fuzz instance in the following way to prevent non-determinism present in the JVM from introduc- 
ing unnecessary noise into our experiments. Every $\mu$ Fuzz instance runs within the EyeVM in interpreted mode in order to maintain consistent run-time measurements for methods under test. If $\mu$ Fuzz runs with JIT enabled, our measurement instrumentation no longer profiles the method under test, but rather the JVM's response to fuzzing the method under test. A JVM with JIT enabled responds by compiling the bytecode that implements the method under test into equivalent native code in the host machine's instruction set architecture and executes it in a separate code cache. This causes the method under test's runtime to change dramatically during microfuzzing and would skew our results. For this reason, we run $\mu$ Fuzz in the EyeVM in interpreted mode to ensure consistent measurements.

Upon receiving a method under test, $\mu$ Fuzz queries the CPUs available by obtaining the process's CPU affinity mask with sched_getaffinity. $\mu$ Fuzz then calls sched_setaffinity to pin the thread running the method under test to the lowest CPU given in the affinity mask. This confines the method under test to a single CPU for the duration of micro-fuzzing and also requires that every $\mu$ Fuzz instance have access to at least two CPUs, one for the method under test, and the remainder for the JVM's own threads.

Each time $\mu$ Fuzz successfully invokes the method under test, it submits a test case for storage in the results database. Every test case generated by $\mu$ Fuzz consists of the input given to the method under test and the number of clock cycles it consumes when invoked on the input. $\mu$ Fuzz interprets exceptions as a signal that an input is malformed, and therefore all such test cases are discarded. Ignoring input that causes the method under test to throw an exception restricts $\mu$ Fuzz's search space to that of valid inputs while it attempts to maximize resource consumption. In a different context, these test cases could be considered a potential attack vector for triggering DoS, but not due to an AC vulnerability.

\section{Evaluation}

In this section, we describe an evaluation of our prototype implementation of HotFuzz. This evaluation focuses on the testing procedure's efficiency in finding AC vulnerabilities in Java libraries, and additionally considers the effect of seed input instantiation strategy on micro-fuzzing efficiency. In particular, we define the performance of micro-fuzzing as the number of $\mathrm{AC}$ vulnerabilities detected in a test artifact over time, and consider one strategy to outperform another if the strategy detects more AC vulnerabilities given the same time budget. In accordance with recently proposed guidelines for evaluating new fuzz testing techniques [33], we evaluate our proposed seed selection strategy (SRI) by comparing the performance of micro-fuzzing with SRI-based seeds to microfuzzing with "empty seed values" (IVI-based seeds).

To the best of our knowledge, including existing fuzzers in our evaluation that adapt AFL [49] and libFuzzer [31] to Java programs to detect $\mathrm{AC}$ vulnerabilities would require significant engineering effort both in terms of instrumentation and designing test harnesses around our artifacts. Furthermore, the results those tools have achieved on real world code bases like the JRE or Java libraries appear limited to individual methods and find bugs that crash the method under test with a thrown exception, or individual challenges from the STAC program. For these reasons, we exclude those tools from our evaluation.

We evaluate HotFuzz over the Java Runtime Environment (JRE), all challenge programs developed by red teams in the DARPA Space and Time Analysis for Cybersecurity (STAC) program, and the 100 most popular libraries available on the Maven repository. This set of evaluation artifacts presents the opportunity to detect previously unknown vulnerabilities in real-world software as well as to validate HotFuzz on programs for which we have ground truth for AC vulnerabilities.

For the real-world software evaluation, we selected the JRE as it provides basic functionality utilized by every Java program. Given Java's widespread deployment across domains that range from embedded devices to high performance servers, any unknown AC vulnerabilities in the JRE present significant security concerns to programs that utilize those methods. For this reason, we evaluate HotFuzz over all methods in the JRE in order to measure its ability to detect unknown AC vulnerabilities in production software. Specifically, we consider JRE 1.8.0_181 from Java 8 as a library under test in our evaluation.

In addition to the JRE, Java programs frequently rely on libraries available through the popular Maven Repository, which as of 2019 hosts 15.1 million artifacts. While this repository provides a convenient way to download an application's library dependencies, it also introduces any unknown vulnerabilities hiding within them into an application. In order to understand how vulnerable Maven's libraries are to AC attacks, we evaluate HotFuzz over the repository's 100 most popular libraries. A library's popularity on Maven is defined by the number of artifacts that include it as a dependency. For every Maven library we consider in our evaluation, we micro-fuzz every method contained in the library, and exclude the methods contained in its dependencies.

a) Findings Summary: In conducting our evaluation, HotFuzz detected previously unknown AC vulnerabilities in the JRE, Maven Libraries, and discovered both intended and unintended AC vulnerabilities in STAC program challenges. Section V-A documents the experimental setup used to obtain these findings. Section V-B summarizes how the seed input generation strategy impacts micro-fuzzing performance, and provides several detailed case studies of micro-fuzzing results for the JRE, STAC challenges, and Maven libraries.

\section{A. Experimental Set Up}

We implement HotFuzz as a distributed system running within an on-premise Kubernetes cluster. The cluster consists of 64 CPUs and 256 GB of RAM across 6 Dell PowerEdge R720 Servers with Intel Xeon $2.4 \mathrm{GHz}$ Processors. To microfuzz a given library under test, we deploy a set of $\mu$ Fuzz instances onto the cluster that consume individual methods from a message broker.

For each individual method under test, each $\mu$ Fuzz instance creates an initial population of inputs to the method, and runs a genetic algorithm that searches for inputs that cause the method under test to consume the most execution time. In addition to the method under test, every job submitted to HotFuzz requires configuration parameters given in Table I.

Recall that HotFuzz makes no assumptions about the code under test, and therefore it is critical to configure timeouts at each step of this process in order for the whole system 
TABLE I: The parameters given to every $\mu$ Fuzz instance. Multiple timeouts prevent HotFuzz from stopping because of individual methods that may be too difficult to micro-fuzz efficiently.

\begin{tabular}{llr}
\hline Parameter & Definition \\
\hline$\alpha$ & The maximum value SRI will assign to a primitive type when constructing an object & Value \\
$\psi$ & The maximum amount of time to create the initial population & 256 \\
$\lambda$ & The time that may elapse betwen measuring the fitness of two method inputs & $5 \mathrm{~s}$ \\
$\omega$ & The amount of time required for a method to run in order to generate an AC witness \\
$\gamma$ & The wall clock time limit for the GA to evaluate the method under test. & \\
$\pi$ & The size of the initial population & $60 \mathrm{~s}$ \\
$\chi$ & The probability two parents produce offsprint in a given generation \\
$\tau$ & The probability an individual mutates in a generation & 100 \\
$\epsilon$ & The percent of the most fit individuals that carry on to the next generation \\
$\nu$ & The number of generations to run the GA & 0.5 \\
$\sigma$ & The amount of time required for an AC witness to run outside the analysis framework in JIT mode in order to confirm it & 5s \\
\hline
\end{tabular}

to complete for all methods in a library under test. For this reason, the parameters $(\psi, \lambda, \omega, \gamma)$ configured various timeouts that ensured HotFuzz ran end to end within a manageable time frame. This is important in order to prevent problems caused by fuzzing individual methods or calling specific constructors from halting the entire micro-fuzzing pipeline. We determined the values for these parameters empirically, and only added each parameter after we observed a need for each one in our evaluation. The parameters $(\pi, \chi, \tau, \epsilon, \nu)$ configured the genetic algorithm (GA) within HotFuzz. In our evaluation, we assigned these parameters the initial values recommended for genetic algorithm experiments [22]. Finally, $\sigma$ configured the timeout used in the witness validation stage. Observe that we configured $\sigma$, the time required to confirm a witness as an $\mathrm{AC}$ vulnerability, to be half of $\omega$, the time needed to detect a witness. Our intuition behind this choice is that a given test case will run much faster with JIT enabled than in our interpreted analysis environment, and hence the runtime required to confirm a witness is lower than the time required to detect it. We use the same parameters for every method under test and do not tune these parameters for specific methods. We argue that this provides a generic approach to detecting AC vulnerabilities in arbitrary Java methods.

To micro-fuzz each library under test, we created a pair of fuzzing jobs with identical parameters for each method contained in the library with the exception of the $\alpha$ parameter. Each pair consisted of one job that used the IVI seed input generation strategy, and the other used the SRI strategy with the $\alpha$ parameter which bounds the values used when constructing the seed inputs for micro-fuzzing. The libraries under test that we consider for our evaluation are all 80 engagement articles given in the STAC program, and every public method contained in a public class found in the JRE and the 100 most popular libraries available on the Maven repository. For the latter, we consider these public library classes and methods as the interface the library reveals to programs that utilize it. Therefore, this provides an ideal attack surface for us to microfuzz for potential $\mathrm{AC}$ vulnerabilities.

a) Seed Input Strategy: Given the definition of SRI presented in Section III-A2b, we use the following procedure to construct the initial seed inputs for every method under test $M$ configured to use SRI in our evaluation. Given the parameter $\alpha, \mu$ Fuzz instantiates a population of seed inputs of size $\pi$ for $M$ as follows. Let $\mathcal{N}(\mu, \sigma)$ be a normal random number distribution with mean $\mu$ and standard deviation $\sigma$. For every primitive type required to instantiate a given class, SRI obtains a value $X \leftarrow \mathcal{N}(0, \alpha / 3)$. This allows micro-fuzzing to favor small values centered around 0 that exceed the chosen parameter $\alpha$ with small probability. To be precise, configuring $\alpha$ to be three times the size of the standard deviation $\sigma$ of our random number distribution $\mathcal{N}$ makes $\operatorname{Pr}(|X|>\alpha)<0.135 \%$. This ensures that the primitive values we use to instantiate objects stay within the range $[-\alpha, \alpha]$ with high probability.

\section{B. Experimental Results}

In our evaluation, HotFuzz detected 26 previously unknown AC vulnerabilities in the Java 8 JRE, detects both intended and unintended vulnerabilities in challenges from the STAC program, and detects $132 \mathrm{AC}$ vulnerabilities in 47 libraries from the 100 most popular libraries found on Maven. Table II breaks down both the total wall-clock time HotFuzz spent micro-fuzzing the JRE, STAC engagement challenges, and Maven libraries for each seed selection strategy and reports micro-fuzzing's throughput measured by the average number of test cases produced per hour. We define a test case to be a single input generated by HotFuzz for a method under test. Overall, micro-fuzzing with SRI-derived seed inputs required more time to micro-fuzz the artifacts in our evaluation, but also produced more test cases overall.

1) Impact of Seed Input Generation Strategy: Table II presents the results of micro-fuzzing the JRE, all the challenges contained in the DARPA STAC program, and the 100 most popular libraries available on Maven using both IVI and SRIderived seed inputs. Overall, micro-fuzzing with both strategies managed to invoke $29.17 \%$ of the methods contained in the JRE, $32.96 \%$ of the methods given in the STAC program, and $29.66 \%$ of the methods found in the 100 most popular Maven libraries. As the results indicate, neither seeding strategy is categorically superior to the other, although SRI does consistently outperform IVI on each of the artifacts included in our evaluation. For example, when considering the results over the JRE, SRI identifies 13 vulnerabilities that IVI does not, compared to the 8 vulnerabilities IVI finds that SRI does not. At the same time, both strategies are able to find another 5 vulnerabilities. We observe the same pattern in both the STAC artifacts and Maven libraries we included in our evaluation, although observe that both strategies were able to solve the same number of challenges in our experiment over the STAC articles (see Case Study V-B4). These results indicate that SRI outperforms IVI as a seed input strategy, and overall the two approaches are complementary. Furthermore, their combined results show that relatively simple micro-fuzzing can expose serious availability vulnerabilities in widely-used software. 


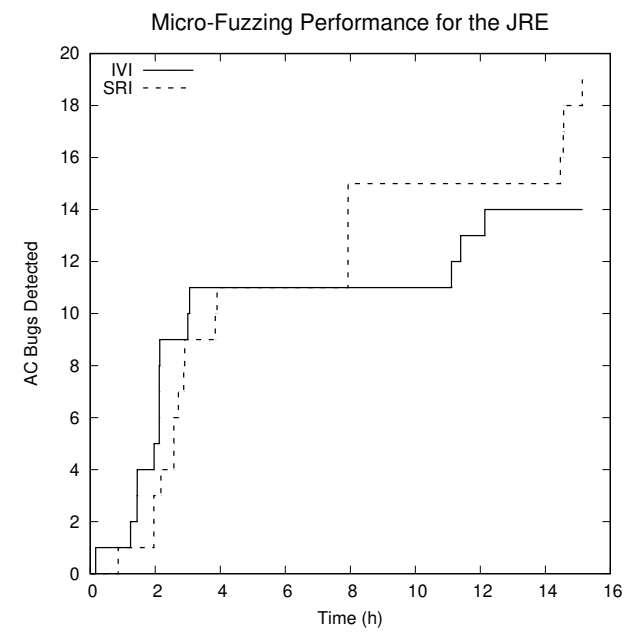

(a)

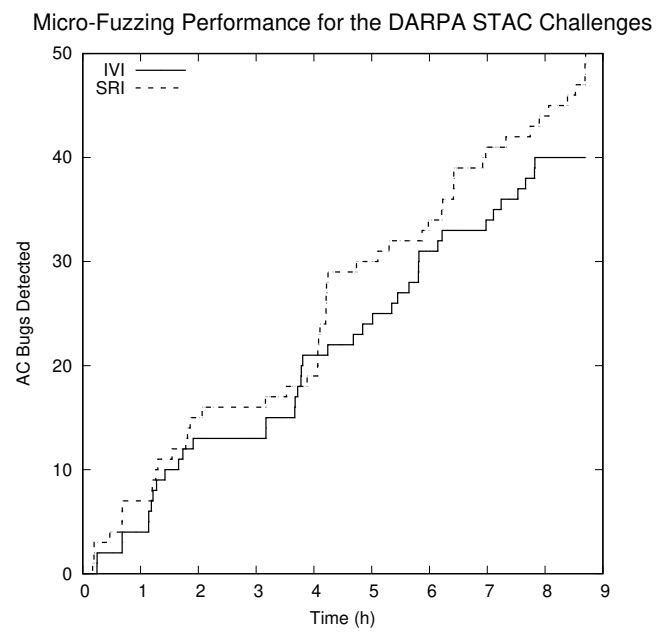

(b)

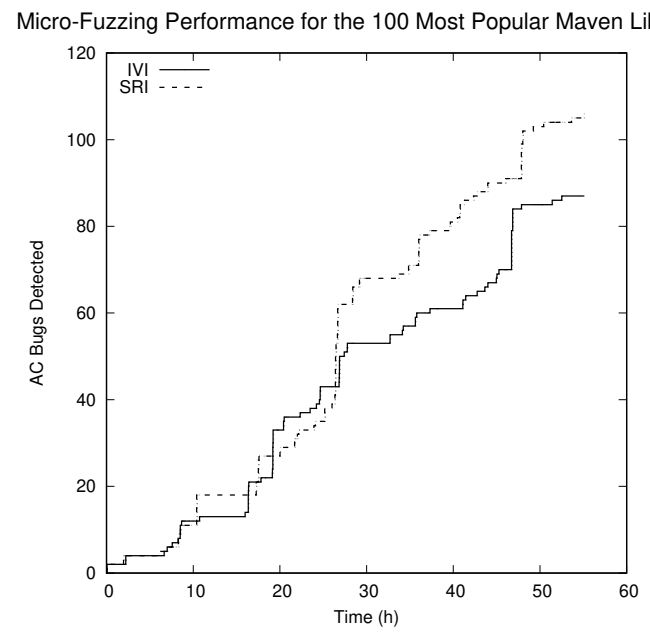

(c)

Fig. 2: Measuring HotFuzz's performance while micro-fuzzing the Java Runtime Environment (JRE) (Figure 2a), all of the challenges given in the DARPA Space and Time Analysis for Cybersecurity (STAC) program (Figure 2b), and the 100 Most Popular Libraries found in Maven [8] (Figure 2c) using both IVI and SRI-derived seed inputs. Observe that micro-fuzzing with SRI-derived seed inputs outperforms IVI-derived seed inputs for every artifact contained in our evaluation.

Figure 2 visually compares the performance of micro-fuzzing the JRE, STAC challenges, and the 100 most popular Maven libraries, using both IVI and SRI-derived seed inputs. From these results, we see that SRI-derived seed inputs produce a marginal improvement over IVI inputs, as micro-fuzzing with SRI-derived seed inputs detects more AC bugs than IVI seed inputs in each of our evaluation artifacts.

Figure 3 provides a visual comparison between IVI and SRI-based micro-fuzzing on a method provided by the JRE that works on regular expressions. According to the documentation, the RE.split (String input) method splits a given string input into an array of strings based on the regular expression boundaries expressed in the compiled regular expression instance RE. Figure 3a shows how microfuzzing this method using IVI-based seeds fails to arrive at a test case that demonstrates the vulnerability. In contrast,
Figure $3 \mathrm{~b}$ shows how using SRI-based seed inputs allows HotFuzz to detect the vulnerability. Additionally, we note that micro-fuzzing with SRI-derived seed inputs requires fewer test cases than micro-fuzzing with IVI-based seeds. When we traced the execution of the exploit found by HotFuzz in the EyeVM in tracing mode, we discovered that the method called the StringCharacterIterator.isEnd method from the com.sun.org.apache.regexp.internal package with alternating arguments indefinitely. We observed the PoC produced by HotFuzz run for 7 days on a Debian system with Intel Xeon E5-2620 CPUs before stopping it. We have disclosed our PoC to Oracle and await their response.

Our evaluation revealed a second AC vulnerability within the same $R E$ package called subst(String substituteIn, String substitution) that substitutes every occurrence of the compiled regular expression 
TABLE II: A comparison of fuzzing outcomes when evaluating HotFuzz on Java libraries using IVI and SRI seed inputs.

\begin{tabular}{|c|c|c|c|c|c|c|c|c|c|c|c|c|c|c|}
\hline \multirow[t]{2}{*}{ Library } & \multirow{2}{*}{$\frac{\text { Size }}{\text { No. Methods }}$} & \multicolumn{3}{|c|}{ AC Witnesses Detected } & \multicolumn{3}{|c|}{ AC Witnesses Confirmed } & \multicolumn{3}{|c|}{ Methods Covered } & \multicolumn{2}{|c|}{ Fuzzing Time (hrs) } & \multicolumn{2}{|c|}{ Throughput (tests/hr) } \\
\hline & & Both & IVI & SRI & Both & IVI & SRI & Both & IVI & SRI & IVI & SRI & IVI & SRI \\
\hline$J R E$ & 91632 & 6 & 8 & 13 & 5 & 8 & 13 & 23818 & 2780 & 1439 & 14.3 & 19.5 & 4389675 & 3092866 \\
\hline DARPA STAC & 67494 & 34 & 6 & 15 & 5 & 0 & 0 & 8064 & 847 & 1162 & 7.8 & 8.7 & 3608741 & 3172502 \\
\hline
\end{tabular}

in substituteIn with the string substitution. After tracing the PoC produced by HotFuzz, we observed that it has the same underlying problem as RE.split. That is, it appears to loop indefinitely checking the result of StringCharacterIterator.isEnd. We observed the PoC for RE. subst running for 12 days on the same Debian system on which we tested the RE.split PoC before stopping it. After reporting the issue to Oracle, they claimed it is not a security issue since the RE. subst method is protected and an attacker would have to perform a non-trivial amount of work to access it. That being said, the test case generated by HotFuzz is only 579 bytes in size and no method in the OpenJDK sources utilizes the RE.subst method outside of the OpenJDK test suite. This method appears to serve no purpose beyond providing a potential attack surface for DoS.

2) Case Study: Detecting AC Vulnerabilities with IVI-based Inputs: Our evaluation revealed the surprising fact that 6 methods in the JRE contain AC vulnerabilities exploitable by simply passing empty values as input. Figure 6 shows the 6 utilities and APIs that contain AC vulnerabilities that an adversary can exploit. Upon disclosing our findings to Oracle they communicated that five of the six methods (lines 1-29) belong to internal APIs and that no path exists for malicious input to reach them. They recognized DecimalFormat's behavior (lines 31-34) as a functional bug that they will fix in an upcoming release. Oracle's assessment assumes that a malicious user will not influence the input of the public DecimalFormat constructor. Unless programs exclusively pass string constants to the constructor as input, this is a difficult invariant to always enforce.

3) Case Study: Arithmetic DoS in Java Math: As a part of our evaluation, HotFuzz detected 5 AC vulnerabilities inside the JRE's Math package. To the best of our knowledge, no prior CVEs document these vulnerabilities. We developed proofof-concept exploits for these vulnerabilities and verified them across three different implementations of the JRE from Oracle, IBM, and Google. The vulnerable methods and classes provide abstractions called BigDecimal and BigInteger for performing arbitrary precision arithmetic in Java. Any Java program that performs arithmetic over instances of BigDecimal derived from user input may be vulnerable to AC exploits, provided an attacker can influence the value of the number's exponent when represented in scientific notation.

A manually defined exploit on BigDecimal . add in Oracle's JDK (Versions 9 and 10) can run for over an hour even when Just-in-Time (JIT) compilation is enabled. On IBM's J9 platform, the exploit ran for 4 and a half months, as measured by the time utility, before crashing. When we exploit the vulnerability on the Android 8.0 Runtime (ART), execution can take over 20 hours before it ends with an exception when run inside an x86 Android emulator.
We reported our findings to all three vendors and received varying responses. IBM assigned a CVE [2] for our findings. Oracle considered this a Security-in-Depth issue and acknowledged our contribution in their Critical Patch Update Advisory [9]. Google argued that it does not fall within the definition of a security vulnerability for the Android platform.

HotFuzz automatically constructs valid instances of BigDecimal and BigInteger that substantially slow down methods in both classes. For example, simply incrementing 1 e2147483647 by 1 takes over an hour to compute on Oracle's JDK even with Just-in-Time (JIT) Compilation enabled. HotFuzz finds these vulnerabilities without any domain-specific knowledge about the Java Math library or the semantics of its classes; HotFuzz derived all instances required to invoke methods by starting from the BigDecimal constructors given in the JRE.

The underlying issue in the JRE source code that introduces this vulnerability stems from how it handles numbers expressed in scientific notation. Every number in scientific notation is expressed as a coefficient multiplied by ten raised to the power of an exponent. The performance of arithmetic over these numbers in the JRE is sensitive to the difference between two numbers' exponents. This makes addition over two numbers with equal exponents, such as 1e2147483647 and $2 e 2147483647$, return immediately, whereas adding $1 e 2147483647$ to $1 e 0$, can run for over an hour on Oracle's JVM.

The root cause of this performance overhead lies in how the JDK transforms numbers during its arithmetic operations [1]. For example, suppose a program uses the BigDecimal class to compute the sum $x_{1} \times 10^{y_{1}}+x_{2} \times 10^{y_{2}}$ where $y_{1} \neq y_{2}$. Let $x_{\min }$ be the coefficient that belongs to the smaller exponent and $x_{\max }$ the coefficient that belongs to the larger exponent. The add method first computes the difference $\left|y_{1}-y_{2}\right|$ and then defines an integer $x_{\text {scaled }}$, an instance of BigInteger which may represent an integer of arbitrary size, and directly computes $x_{\text {scaled }}=x_{\max } \times 10^{\left|y_{1}-y_{2}\right|}$. This allows the add method to complete the addition by returning a BigDecimal represented with a coefficient given by $x_{\text {scaled }}+x_{\min }$ and an exponent given by the smaller of $y_{1}$ and $y_{2}$. Unfortunately, it also opens up the possibility of a significant performance penalty while computing $x_{\text {scaled }}$ that an adversary could exploit to achieve DoS when the difference between $y_{1}$ and $y_{2}$ is large. In the $\mathrm{PoC}$ given above, add must compute $x_{\text {scaled }}=1 \times 10^{2147483647}$ before simply adding it to 1 with an exponent of 0. Anecdotally, the EyeVM helped pinpoint this issue by tracing the execution of the PoC. When tracing $1+1 \times 10^{2147483647}$, the method bigMultiplyPowerTen started computing $1 \times 10^{2147483647}$ but did not return before we manually stopped the PoC. This method appeared to be the source of the performance penalty because it was absent when 


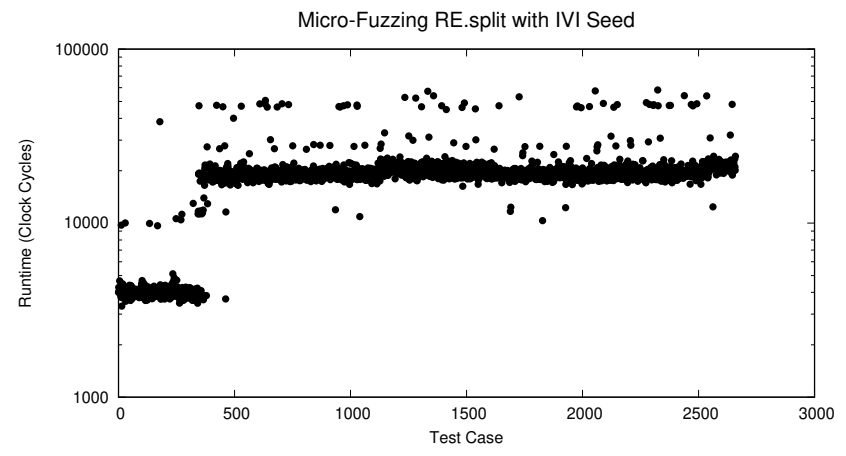

(a) IVI-derived inputs.

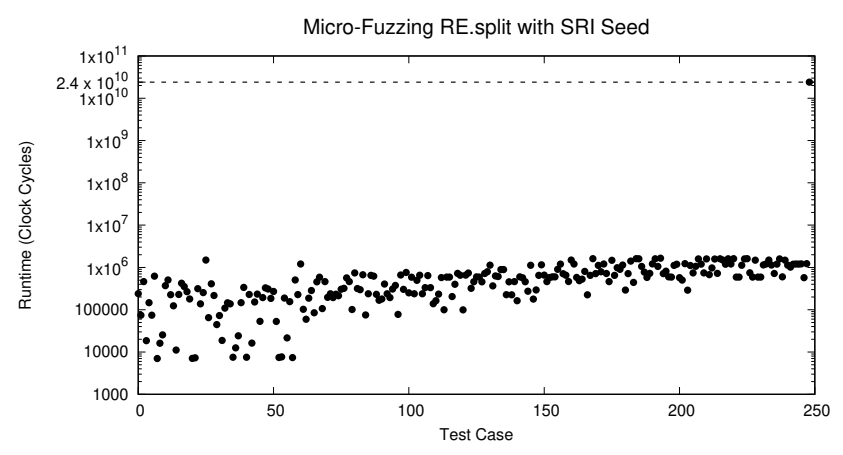

(b) SRI-derived inputs.

Fig. 3: Visualization of micro-fuzzing com.sun.org.apache.regexp.internal.RE.split(String input) with IVI and SRI-derived inputs. Micro-fuzzing with IVI-derived inputs fails to detect a zero-day AC vulnerability in the JRE, while SRI-derived inputs detect the vulnerability correctly. Observe that the y-axis for graph a is 5 orders of magnitude smaller than graph $\mathrm{b}$. Graph $\mathrm{b}$ shows the test case that triggers the $\mathrm{AC}$ vulnerability in the upper-right-hand corner.

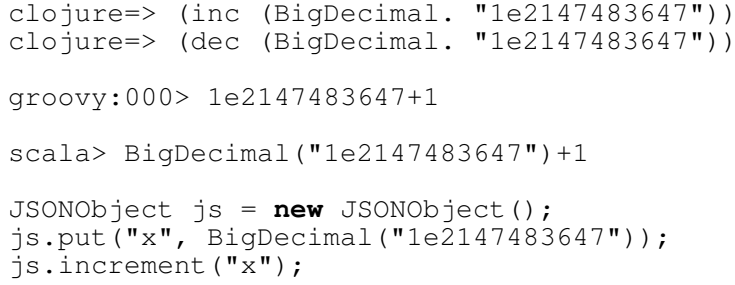

Fig. 4: Proof of concept exploits that trigger inefficient arithmetic operations for the BigDecimal class in Clojure, Scala, Groovy, and the org.json library.

tracing $2 \times 10^{2147483647}+1 \times 10^{2147483647}$ which completed immediately.

After observing this result, we surveyed popular libraries that use BigDecimal internally, and developed proof of concepts that exploit this vulnerability as shown in Figure 4. We found that several general purpose programming languages hosted on the JVM are vulnerable to this attack along with org.json, a popular JSON parsing library.

Developers face numerous security threats when they validate input values given as strings. The vulnerabilities we discussed in this section are especially problematic because malicious input is perfectly valid, albeit very large, floating point numbers. If a program performs any arithmetic over a BigDecimal object derived from user input, then it must take care to prevent the user from providing arbitrary numbers in scientific notation. Likewise, these results show that developers must be careful when converting between these two classes, as interpreting certain floating point numbers as integers could suddenly halt their application. This complicates any input validation that accepts numbers given as strings. Our results reveal that failure to implement such validation correctly could allow remote adversaries to slow victim programs to a halt.
After we disclosed this vulnerability to vendors, we observed that recent releases of the JRE provide mitigations for it. For example, in JRE build 1.8.0_242, the PoCs we present in this section immediately end with an exception thrown by the BigInteger class. Instead of naively computing $x_{\text {scaled }}$, the new implementation first checks to see if $x_{\text {scaled }}$ will exceed a threshold and, if so, aborts the inefficient computation with an exception before it can affect the availability of the overall process. While this defends against the original AC vulnerability, it also introduces a new opportunity for DoS by allowing an adversary to trigger exceptions that programs may fail to properly handle.

4) Case Study: DARPA STAC Challenges: The DARPA Space and Time Analysis for Cybersecurity (STAC) program contains a set of challenge programs developed in Java that test the ability of program analysis tools to detect AC vulnerabilities. In this case study, we measure HotFuzz's ability to automatically detect $\mathrm{AC}$ vulnerabilities found in these challenges. We began by feeding the challenges into HotFuzz which produced a corpus of test cases that exploit AC vulnerabilities contained in the challenges.

However, these test cases on their own do not answer the question of whether a given challenge is vulnerable to an $\mathrm{AC}$ attack, because challenges are whole programs that receive input from sources such as standard input or network sockets, and HotFuzz detects AC vulnerabilities at the method level. Therefore, given a challenge that is vulnerable to an AC attack, we need a way to determine whether one of its methods that HotFuzz marks as vulnerable is relevant to the intended vulnerability.

The STAC challenges provide ground truth for evaluating HotFuzz in the form of proof-of-concept exploits on challenges with intended vulnerabilities. We define the following procedure to assess whether HotFuzz can detect an AC vulnerability automatically. We start by executing each challenge that contains an intended vulnerability in the EyeVM in tracing mode, 


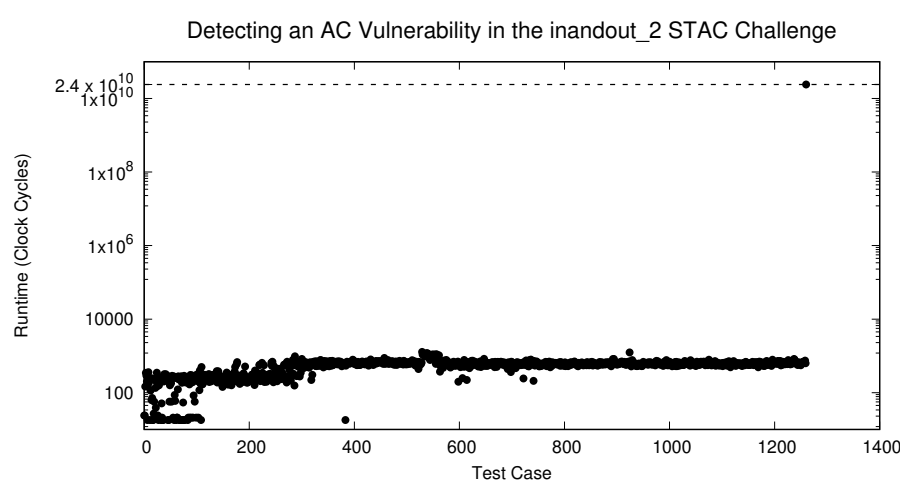

Fig. 5: Visualization of HotFuzz detecting an AC vulnerability found inside the inandout_2 challenge in the DARPA STAC Program. The test case that triggers the AC vulnerability in the challenge can be found in the upper-right-hand corner.

and execute the challenge's exploit on the running challenge. This produces a trace of every method invoked in the challenge during a successful exploit. If HotFuzz marks a method $M$ as vulnerable in the output for challenge $C$, and $M$ appears in the trace for $C$, we count the challenge as confirmed.

When we conducted this experiment on all the challenges contained in the STAC program vulnerable to AC attacks, we found that HotFuzz automatically marked 5 out of 36 challenges as vulnerable. One challenge, inandout_2 provides a web service that allows users to order pizzas online. By running HotFuzz over this challenge, it identifies multiple methods with AC vulnerabilities in its code. Figure 5 visualizes HotFuzz detecting an AC vulnerability in the PizzaParameters.subsequentEnergyof2 (int) method found in the challenge. When we traced the execution of an exploit that achieved DoS against the pizza service, we observed the vulnerable method subsequentEnergyOf2 identified by HotFuzz in the exploit's trace.

5) Case Study: Slow Parsing in org.json: Over the course of our evaluation HotFuzz detected a previously unknown AC vulnerability inside the popular org.json library. The vulnerable method, JSONML.toJSONObject (String) converts an XML document represented as a string into an equivalent JSONObject. This method is public, and instructions for its use in programs can be found in tutorials online [6]. Given the popularity of the org.json library on Maven, application developers may unknowingly expose themselves to DoS attacks by simply parsing XML strings into JSON.

Our experimental results obtained by micro-fuzzing the org. json library also demonstrated the utility of using SRI seed inputs over IVI seed inputs. Over the course of our evaluation, test cases evolved from IVI seed inputs failed to successfully invoke the toJSONOb ject method after 4,654 test cases. Meanwhile, the 96th SRI-derived seed input successfully triggered the vulnerability. The second stage of our pipeline successfully validated this SRI test case represented as a 242 character string. After our evaluation completed, we

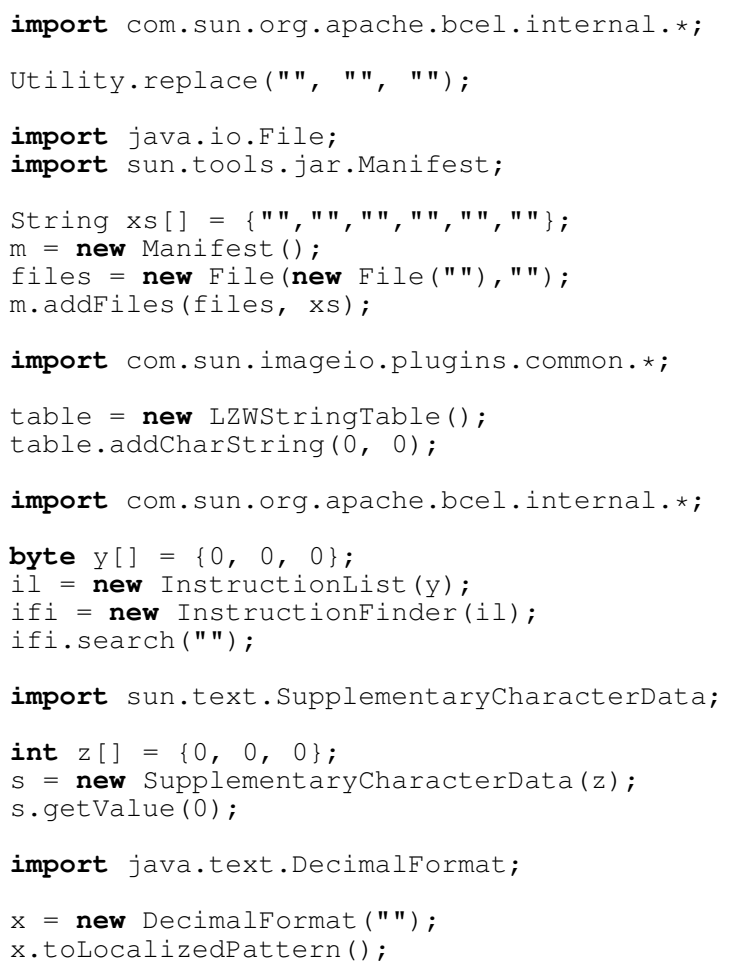

Fig. 6: Proof of concept exploits for AC vulnerabilities that require only IVI-based inputs to trigger.

took the PoC program generated by HotFuzz and observed it running for 120 hours on a Debian system with Intel Xeon E5-2620 CPUs in an unmodified Java environment with JIT enabled. The SRI strategy that produced this test case sampled primitive values uniformly at random from the interval $[0, \alpha)$. Sampling from the normal distribution $\mathcal{N}(0, \alpha / 3)$ detected a bug in the JSONML package, but the test case did not pass the witness validation stage.

During our evaluation, HotFuzz started with no prior knowledge about org.json, JSON, or XML. Nonetheless, after simply passing the org.json library to HotFuzz as input and microfuzzing its methods using SRI-derived seed inputs, we were able to uncover a serious AC vulnerability that exposes programs that depend on this library to potential DoS attacks. We communicated our findings to the owners of the JSON-java project [5] who confirmed the behavior we observed as a bug.

The developers immediately found the root cause of the infinite loop after debugging the method starting with the test case produced by HotFuzz. This test case opened an XML comment with the string $<$ ! which prompted a loop inside the toJSONObject method to check for special characters that represent the beginning $(<)$ and end $(>)$ of a comment until it reached the end of the original comment. The test case produced by HotFuzz caused a method in this loop, nextMeta, to always return the same character, and therefore prevented the loop from advancing. After fixing this bug, the developers included our test case in org.json's test suite in order to prevent the issue from occurring in future releases. The test case that triggered this infinite loop is small ( 242 bytes) and demonstrates the potential micro-fuzzing has 
to uncover serious AC vulnerabilities hiding in popular Java libraries. After micro-fuzzing the toJSONOb ject method on the patched org. json library, we discovered 6 test cases that triggered $\mathrm{AC}$ vulnerabilities, but these were fixed in the latest release of org.json.

\section{RELATED WORK}

HotFuzz relates to previous work in four categories: (i) AC Vulnerability Analysis, (ii) Test Case Generation, (iii) Fuzz Testing, and (iv) Resource Analysis.

\section{A. AC Vulnerability Analysis}

Prior work for detecting AC vulnerabilities in Java programs includes static analysis on popular libraries [32], [38], [62], object-graph engineering on Java's serialization facilities [21], and exploiting worst-case runtime of algorithms found in commercial grade networking equipment [20]. On the Android platform, Huang et al. [29] use a combination of static and dynamic analysis to detect AC vulnerabilities within Android's System Server. Further up the application stack, Pellegrino et al. [45] identify common implementation mistakes that make web services vulnerable to DoS attacks. Finally, Holland et al. [28] proposes a 2 stage analysis for finding AC vulnerabilities.

Prior work for detecting AC vulnerabilities is custom-tailored to specific domains (e.g., serialization, regular-expression engines, Android Services, or web applications) and therefore often requires human assistance. HotFuzz differs from these approaches in that it is generically applicable to any Java program without human intervention, intuition, or insight.

\section{B. Test Case Generation}

Program analysis tools can generate test cases that exercise specific execution paths in a program and demonstrate the presence of bugs. Several tools perform symbolic execution within the Java Pathfinder [26] platform [30], [39], [65] in order to increase code coverage in Java test suites. Symbolic execution has found serious security bugs when applied to whole programs [16], [42] and in under-constrained settings [48] similar to HotFuzz. Toffola et al. [60] introduced PerfSyn which uses combinatoric search to construct test programs that trigger performance bottlenecks in Java methods.

\section{Fuzz Testing}

State of the art fuzzers [7], [64] combine instrumentation on a program under test to provide feedback to a genetic algorithm that mutates inputs in order to trigger a crash. Active research topics include deciding optimal fuzzing seeds [51] and techniques for improving a fuzzer's code coverage [17], [61]. Prior work has seeded fuzzing by replaying sequences of kernel API calls [25], commands from Android apps to smart IoT Devices [18], and input provided by human assistants [55]. Recent techniques for improving code coverage during fuzz testing include introducing selective symbolic execution [58], control- and data-flow analysis on the program under test [50], reducing collisions in code coverage measurements [23], and altering the program under test [46]. Prior work applies existing fuzz testers to discover AC vulnerabilities in whole programs [37], [47], and in Java programs by combining fuzz testing with symbolic execution [43] or seeding black box fuzzing with information taken from program traces [40]. In contrast, HotFuzz micro-fuzzes individual methods and uses a genetic algorithm on individual Java objects in order to find inputs to these methods that demonstrate the presence of $\mathrm{AC}$ vulnerabilities. This departs from prior approaches that restrict fuzzing inputs to flat bitmaps.

\section{Resource Analysis}

Recent interest in AC and side-channel vulnerabilities increased the focus on resource analysis research. In this area, Proteus [63] presented by Xie et al. and Awadhutkar et al. [13] study sensitive paths through loops that might represent AC vulnerabilities. Meanwhile, Kothary [34], [53] investigates human-machine interaction to improve program analysis for finding critical paths and side channels. In Comb [27], Holland et al. investigate how to improve computation of all relevant program behaviors.

Other resource-oriented static analyses have also been proposed [15], [59]. This line of work is based on statically inferred properties of programs and their resource usage. In contrast, HotFuzz provides quantitative measurements of program behavior over concrete inputs in a dynamic, empirical fashion.

\section{CONCLUSION}

In this work, we present HotFuzz, a fuzzer that detects Algorithmic Complexity (AC) vulnerabilities in Java libraries through a novel approach called micro-fuzzing. HotFuzz uses genetic optimization of test artifact resource usage seeded by Java-specific Identity Value and Small Recursive Instantiation (IVI and SRI) techniques to search for inputs that demonstrate $\mathrm{AC}$ vulnerabilities in methods under test. We evaluate HotFuzz on the Java 8 Runtime Environment (JRE), challenge programs developed in the DARPA Space and Time Analysis for CyberSecurity (STAC) program, and the 100 most popular libraries on Maven. In conducting this evaluation, we discovered previously unknown $\mathrm{AC}$ vulnerabilities in production software, including 26 in the JRE, 132 in 47 Maven Libraries, as well as both known and unintended vulnerabilities in STAC evaluation artifacts. Our results demonstrate that the array of testing techniques introduced by HotFuzz are effective in finding AC vulnerabilities in real-world software.

\section{ACKNOWLEDGEMENTS}

This work was partially supported by the National Science Foundation (NSF) under grant CNS-1703454 award, ONR grants N00014-19-1-2364 and N00014-17-1-2011, and Secure Business Austria. The views and conclusions contained herein are those of the authors and should not be interpreted as representing the official policies or endorsements, either expressed or implied, of any government agency.

\section{REFERENCES}

[1] Arithmetic in JDK BigDecimal. https://hg.openjdk.java.net/ jdk8u/jdk8u-dev/jdk/file/d13abc740e42/src/share/classes/java/math/ BigDecimal.java\#14464.

[2] CVE-2018-1517. http://cve.mitre.org/cgi-bin/cvename.cgi?name= CVE-2018-1517.

[3] CVE-2018-5390. https://nvd.nist.gov/vuln/detail/CVE-2018-5390\# vulnCurrentDescriptionTitle.

[4] CVE-2019-6486. http://cve.mitre.org/cgi-bin/cvename.cgi?name= CVE-2019-6486.

[5] JSON-Java Project. https://stleary.github.io/JSON-java/.

[6] JSONML Tutorials Point. https://www.tutorialspoint.com/org_json/org_ json_jsonml.htm. 
[7] libFuzzer - A library for coverage-guided fuzz testing. https://llvm.org/ docs/LibFuzzer.html.

[8] Maven Repository. https://mvnrepository.com/.

[9] Oracle Critical Patch Update Advisory - January 2019. https://www. oracle.com/technetwork/security-advisory/cpujan2019-5072801.html.

[10] The Java Virtual Machine Specification. https://docs.oracle.com/javase/ specs/jvms/se8/html/index.html.

[11] The JVM Tool Interface (JVM TI): How VM Agents Work. https: //www.oracle.com/technetwork/articles/javase/index-140680.html.

[12] Aschermann, C., Schumilo, S., Blazytko, T., Gawlik, R., AND Holz, T. Redqueen: Fuzzing with input-to-state correspondence. In NDSS (2019).

[13] Awadhutkar, P., Santhanam, G. R., Holland, B., and KOTHARI, S. Intelligence amplifying loop characterizations for detecting algorithmic complexity vulnerabilities. In 2017 24th AsiaPacific Software Engineering Conference (APSEC) (Dec. 2017), vol. 00, pp. 249-258.

[14] Behrens, S., AND Payne, B. Starting the avalanche: Application ddos in microservice architectures.

[15] Carbonneaux, Q., Hoffmann, J., Reps, T. W., and Shao, Z. Automated resource analysis with coq proof objects. In Computer Aided Verification - 29th International Conference, CAV 2017, Heidelberg, Germany, July 24-28, 2017, Proceedings, Part II (2017), pp. 64-85.

[16] Cha, S. K., Avgerinos, T., Rebert, A., And Brumley, D. Unleashing Mayhem on Binary Code. In IEEE Symposium on Security and Privacy, SP 2012, 21-23 May 2012, San Francisco, California, USA (2012), pp. $380-394$

[17] Cha, S. K., Woo, M., And Brumley, D. Program-Adaptive Mutational Fuzzing. In 2015 IEEE Symposium on Security and Privacy, SP 2015, San Jose, CA, USA, May 17-21, 2015 (2015), pp. 725-741.

[18] Chen, J., Diao, W., Zhao, Q., Zuo, C., Lin, Z., Wang, X., Lau, W. C., Sun, M., YANG, R., AND Zhang, K. Iotfuzzer: Discovering memory corruptions in iot through app-based fuzzing. In 25th Annual Network and Distributed System Security Symposium, NDSS 2018, San Diego, California, USA, February 18-21, 2018 (2018).

[19] Crosby, S. A., And Wallach, D. S. Denial of Service via Algorithmic Complexity Attacks. In Proceedings of the 12th USENIX Security Symposium, Washington, D.C., USA, August 4-8, 2003 (2003).

[20] Czubak, A., And SzymaneK, M. Algorithmic Complexity Vulnerability Analysis of a Stateful Firewall. In Information Systems Architecture and Technology: Proceedings of 37th International Conference on Information Systems Architecture and Technology - ISAT 2016 - Part II (2016), pp. 77-97.

[21] Dietrich, J., JezeK, K., Rasheed, S., TahiR, A., And Potanin, A. Evil Pickles: DoS Attacks Based on Object-Graph Engineering. In 31st European Conference on Object-Oriented Programming, ECOOP 2017, June 19-23, 2017, Barcelona, Spain (2017), pp. 10:1-10:32.

[22] Eiben, A. E., AND Smith, J. E. Introduction to Evolutionary Computing. Natural Computing Series. Springer, 2015.

[23] Gan, S., Zhang, C., Qin, X., Tu, X., Li, K., Pei, Z., and Chen, Z. Collafl: Path sensitive fuzzing. In 2018 IEEE Symposium on Security and Privacy, SP 2018, Proceedings, 21-23 May 2018, San Francisco, California, USA (2018), pp. 679-696.

[24] Godefroid, P. Micro execution. In Proceedings of the 36th International Conference on Software Engineering (2014), ACM, pp. 539-549.

[25] HAN, H., AND CHA, S. K. IMF: inferred model-based fuzzer. In Proceedings of the 2017 ACM SIGSAC Conference on Computer and Communications Security, CCS 2017, Dallas, TX, USA, October $30-$ November 03, 2017 (2017), pp. 2345-2358.

[26] HaVelund, K. Java pathfinder, A translator from java to promela. In Theoretical and Practical Aspects of SPIN Model Checking, 5th and 6th International SPIN Workshops, Trento, Italy, July 5, 1999, Toulouse, France, September 21 and 24 1999, Proceedings (1999), p. 152.

[27] Holland, B., Awadhutkar, P., Kothari, S., Tamrawi, A., AND MATHEWS, J. Comb: Computing relevant program behaviors. In Proceedings of the 40th International Conference on Software Engineering: Companion Proceeedings (New York, NY, USA, 2018), ICSE '18, ACM, pp. 109-112.

[28] Holland, B., Santhanam, G. R., AwadhutKar, P., And KothaRI, S. Statically-informed dynamic analysis tools to detect algorithmic complexity vulnerabilities. In Source Code Analysis and Manipulation (SCAM), 2016 IEEE 16th International Working Conference on (2016), IEEE, pp. 79-84

[29] Huang, H., Zhu, S., Chen, K., And LiU, P. From System Service Freezing to System Server Shutdown in Android: All You Need Is a Loop in an App. In Proceedings of the 22nd ACM SIGSAC Conference on Computer and Communications Security, Denver, CO, USA, October 12-6, 2015 (2015), pp. 1236-1247.

[30] Jayaraman, K., Harvison, D., Ganesh, V., and Kiezun, A. jFuzz: A Concolic Whitebox Fuzzer for Java. In First NASA Formal Methods Symposium - NFM 2009, Moffett Field, California, USA, April 6-8, 2009. (2009), pp. 121-125.

[31] Kersten, R. Kelinci. https://github.com/isstac/kelinci.

[32] Kirrage, J., Rathnayake, A., And Thielecke, H. Static analysis for regular expression denial-of-service attacks. In Proceedings of the International Conference on Network and System Security (NSS) (Madrid, Spain, June 2013)

[33] Klees, G., Ruef, A., Cooper, B., Wei, S., ANd Hicks, M. Evaluating fuzz testing. In Proceedings of the 2018 ACM SIGSAC Conference on Computer and Communications Security, CCS 2018, Toronto, ON, Canada, October 15-19, 2018 (2018), pp. 2123-2138.

[34] Kothari, S., Tamrawi, A., and Mathews, J. Human-machine resolution of invisible control flow? In 2016 IEEE 24th International Conference on Program Comprehension (ICPC) (May 2016), pp. 1-4.

[35] KulEshov, E. Using the asm framework to implement common java bytecode transformation patterns. Aspect-Oriented Software Development (2007)

[36] LAF INTEL. Circumventing Fuzzing Roadblocks with Compiler Transformations. https://lafintel.wordpress.com/.

[37] Lemieux, C., Padhye, R., Sen, K., and Song, D. Perffuzz: automatically generating pathological inputs. In Proceedings of the 27th ACM SIGSOFT International Symposium on Software Testing and Analysis, ISSTA 2018, Amsterdam, The Netherlands, July 16-21, 2018 (2018), pp. 254-265.

[38] Livshits, V. B., AND LAM, M. S. Finding Security Vulnerabilities in Java Applications with Static Analysis. In Proceedings of the 14th USENIX Security Symposium, Baltimore, MD, USA, July 31 - August 5, 2005 (2005).

[39] Luckow, K. S., Dimjasevic, M., Giannakopoulou, D., Howar, F., ISBERner, M., KAHSAI, T., RAKAMARIC, Z., AND RAMAN, V. JDart: A Dynamic Symbolic Analysis Framework. In Tools and Algorithms for the Construction and Analysis of Systems - 22nd International Conference, TACAS 2016, Held as Part of the European Joint Conferences on Theory and Practice of Software, ETAPS 2016, Eindhoven, The Netherlands, April 2-8, 2016, Proceedings (2016), pp. 442-459.

[40] Luo, Q., NAir, A., GRechanik, M., AND Poshyvanyk, D. FOREPOST: finding performance problems automatically with feedbackdirected learning software testing. Empirical Software Engineering 22, 1 (2017), 6-56.

[41] Microsoft. Microsoft security risk detection. https://www.microsoft. com/en-us/security-risk-detection/.

[42] Molnar, D., Li, X. C., And Wagner, D. A. Dynamic Test Generation to Find Integer Bugs in x86 Binary Linux Programs. In 
18th USENIX Security Symposium, Montreal, Canada, August 10-14, 2009, Proceedings (2009), pp. 67-82.

[43] Noller, Y., Kersten, R., And Pasareanu, C. S. Badger: complexity analysis with fuzzing and symbolic execution. In Proceedings of the 27th ACM SIGSOFT International Symposium on Software Testing and Analysis, ISSTA 2018, Amsterdam, The Netherlands, July 16-21, 2018 (2018), pp. 322-332.

[44] Ognawala, S., Hutzelmann, T., Psallida, E., And PRETSCHNER, A. Improving function coverage with munch: a hybrid fuzzing and directed symbolic execution approach. In Proceedings of the 33rd Annual ACM Symposium on Applied Computing (2018), ACM, pp. 1475-1482.

[45] Pellegrino, G., Balzarotti, D., Winter, S., And Suri, N. In the compression hornet's nest: A security study of data compression in network services. In 24th USENIX Security Symposium, USENIX Security 15, Washington, D.C., USA, August 12-14, 2015. (2015), pp. $801-816$.

[46] Peng, H., Shoshitaishvili, Y., And Payer, M. T-fuzz: Fuzzing by program transformation. In 2018 IEEE Symposium on Security and Privacy, SP 2018, Proceedings, 21-23 May 2018, San Francisco, California, USA (2018), pp. 697-710.

[47] Petsios, T., Zhao, J., Keromytis, A. D., and Jana, S. Slowfuzz: Automated domain-independent detection of algorithmic complexity vulnerabilities. In Proceedings of the 2017 ACM SIGSAC Conference on Computer and Communications Security, CCS 2017, Dallas, TX, USA, October 30 - November 03, 2017 (2017), pp. 2155-2168.

[48] Ramos, D. A., AND Engler, D. R. Under-Constrained Symbolic Execution: Correctness Checking for Real Code. In 24th USENIX Security Symposium, USENIX Security 15, Washington, D.C., USA, August 12-14, 2015. (2015), pp. 49-64.

[49] Ranken, G. libFuzzer Java. https:/github.com/guidovranken/ libfuzzer-java.

[50] Rawat, S., Jain, V., Kumar, A., Cojocar, L., Giuffrida, C., AND Bos, H. Vuzzer: Application-aware evolutionary fuzzing. In Proceedings of the Network and Distributed System Security Symposium (NDSS) (2017).

[51] Rebert, A., Cha, S. K., Avgerinos, T., Foote, J., Warren, D., Grieco, G., AND BRumley, D. Optimizing Seed Selection for Fuzzing. In Proceedings of the 23rd USENIX Security Symposium, San Diego, CA, USA, August 20-22, 2014. (2014), pp. 861-875.

[52] Russell, K. B., AND BAK, L. The hotspot serviceability agent: An out-of-process high-level debugger for a java virtual machine. In Proceedings of the 1st Java Virtual Machine Research and Technology Symposium, April 23-24, 2001, Monterey, CA, USA (2001), pp. $117-$ 126.

[53] Santhanam, G. R., Holland, B., Kothari, S., and Ranade, N. Human-on-the-loop automation for detecting software side-channel vulnerabilities. In Information Systems Security (Cham, 2017), R. K. Shyamasundar, V. Singh, and J. Vaidya, Eds., Springer International Publishing, pp. 209-230.

[54] Serebryany, K., Bruening, D., Potapenko, A., and Vyukov, D. Addresssanitizer: A fast address sanity checker. In Presented as part of the 2012 USENIX Annual Technical Conference (USENIX ATC 12) (2012), pp. 309-318

[55] Shoshitaishvili, Y., Weissbacher, M., Dresel, L., Salls, C., WANG, R., KRUEgel, C., AND VignA, G. Rise of the hacrs: Augmenting autonomous cyber reasoning systems with human assistance. In Proceedings of the 2017 ACM Conference on Computer and Communications Security (2017), ACM.

[56] Source, G. O. Oss-fuzz. https://github.com/google/oss-fuzz.

[57] Staicu, C., And Pradel, M. Freezing the web: A study of redos vulnerabilities in javascript-based web servers. In 27th USENIX Security Symposium, USENIX Security 2018, Baltimore, MD, USA, August 1517, 2018. (2018), pp. 361-376.
[58] Stephens, N., Grosen, J., Salls, C., Dutcher, A., Wang, R. Corbetta, J., Shoshitaishvili, Y., Kruegel, C., and Vigna, G. Driller: Augmenting Fuzzing Through Selective Symbolic Execution. Internet Society.

[59] Tizpaz-Niari, S., ČErný, P., Chang, B.-Y. E., SAnkaraNARAYANAN, S., AND TRIVEDI, A. Discriminating traces with time In Tools and Algorithms for the Construction and Analysis of Systems (Berlin, Heidelberg, 2017), A. Legay and T. Margaria, Eds., Springer Berlin Heidelberg, pp. 21-37.

[60] Toffola, L. D., Pradel, M., And Gross, T. R. Synthesizing programs that expose performance bottlenecks. In Proceedings of the 2018 International Symposium on Code Generation and Optimization, CGO 2018, Vösendorf / Vienna, Austria, February 24-28, 2018 (2018), pp. 314-326.

[61] Woo, M., Cha, S. K., Gottlieb, S., And Brumley, D. Scheduling Black-Box Mutational Fuzzing. In 2013 ACM SIGSAC Conference on Computer and Communications Security, CCS'13, Berlin, Germany, November 4-8, 2013 (2013), pp. 511-522.

[62] Wüstholz, V., Olivo, O., Heule, M. J. H., And Dillig, I. Static Detection of DoS Vulnerabilities in Programs that Use Regular Expressions. In Tools and Algorithms for the Construction and Analysis of Systems - 23rd International Conference, TACAS 2017, Held as Part of the European Joint Conferences on Theory and Practice of Software, ETAPS 2017, Uppsala, Sweden, April 22-29, 2017, Proceedings, Part II (2017), pp. 3-20.

[63] Xie, X., Chen, B., Liu, Y., Le, W., And Li, X. Proteus: Computing disjunctive loop summary via path dependency analysis. In Proceedings of the 2016 24th ACM SIGSOFT International Symposium on Foundations of Software Engineering (New York, NY, USA, 2016), FSE 2016, ACM, pp. 61-72.

[64] Zalewski, M. American Fuzzy Lop. http://lcamtuf.coredump.cx/afl/.

[65] ZHU, H. JFuzz: A Tool for Automated Java Unit Testing Based on Data Mutation and Metamorphic Testing Methods. In 2015 Second International Conference on Trustworthy Systems and Their Applications, TSA 2015, Hualien, Taiwan, July 8-9, 2015 (2015), pp. 8-15. 